\title{
The 2dF Galaxy Redshift Survey: spectra and redshifts
}

M atthew Colless, ${ }^{1 \star}$ Gavin Dalton, ${ }^{2}$ Steve Maddox, ${ }^{3}$ Will Sutherland, ${ }^{4}$ Peder Norberg, ${ }^{5}$ Shaun Cole, ${ }^{5}$ Joss B land-Hawthorn, ${ }^{6}$ Terry Bridges, ${ }^{6}$ R ussell Cannon, ${ }^{6}$ Chris Collins, ${ }^{7}$ Warrick Couch, ${ }^{8} \mathrm{~N}$ icholas C ross, ${ }^{4} \mathrm{~K}$ athryn Deel ey, ${ }^{8}$ R oberto De Propris, ${ }^{8}$ Simon P. Driver, ${ }^{4}$ George Efstathiou, ${ }^{9}$ Richard S. Ellis, ${ }^{10}$ Carlos S. Frenk, ${ }^{5}$ K arl Glazebrook, ${ }^{11}$ Carole Jackson, ${ }^{1}$ Ofer L ahav, ${ }^{9}$ Ian L ewis, ${ }^{6}$ Stuart L umsden, ${ }^{12}$ Darren M adgwick, ${ }^{9}$ John A. Peacock, ${ }^{13}$ Bruce A. Peterson, ${ }^{1}$ Ian Price, ${ }^{1}$ M ark Seaborne ${ }^{2}$ and Keith Taylor ${ }^{6,10}$ (the 2dFGRS team)

\footnotetext{
${ }^{1}$ Research School of Astronomy and Astrophysics, The Australian National U niversity, Weston Creek, ACT 2611, Australia

${ }^{2}$ Department of Physics, U niversity of 0 xford, Keble Road, Oxford OX13RH

${ }^{3}$ School of Physics and Astronomy, U niversity of Nottingham, Nottingham NG 7 2RD

${ }^{4}$ School of Physics and Astronomy, University of St Andrews, North Haugh, St Andrews, Fife, KY6 9SS

${ }^{5}$ Department of Physics, U niversity of Durham, South Road, Durham DH $13 \mathrm{LE}$

${ }^{6}$ Anglo-Australian Observatory, PO Box 296, Epping, NSW 2121, Australia

${ }^{7}$ Astrophysics Research Institute, Liverpool J ohn M oores U niversity, Twelve Quays House, Birkenhead L14 ILD

${ }^{8}$ Department of Astrophysics, U niversity of New South Wales, Sydney, NSW 2052, Australia

${ }^{9}$ Institute of Astronomy, U niversity of Cambridge, Madingley Road, Cambridge CB3 OHA

${ }^{10}$ Department of Astronomy, California Institute of Technology, Pasadena, CA 91125, USA

${ }^{11}$ D epartment of Physics and Astronomy, J ohns Hopkins U niversity, Baltimore, M D 21218-2686, USA

${ }^{12}$ Department of Physics, U niversity of Leeds, Woodhouse Lane, Leeds LS2 9J T

${ }^{13}$ Institute for Astronomy, U niversity of Edinburgh, Royal Observatory, Blackford Hill, Edinburgh EH $93 \mathrm{HJ}$
}

Accepted 2001 A ugust 9. Received 2001 July 30

\begin{abstract}
A B STR A C T
The 2dF Galaxy Redshift Survey (2dFGRS) is designed to measure redshifts for approximately 250000 galaxies. This paper describes the survey design, the spectroscopic observations, the redshift measurements and the survey data base. The $2 \mathrm{dFGRS}$ uses the $2 \mathrm{dF}$ multifibre spectrograph on the Anglo-A ustralian Telescope, which is capable of observing 400 objects simultaneously over a $2^{\circ}$ diameter field. The source catal ogue for the survey is a revised and extended version of the A PM galaxy catalogue, and the targets are galaxies with extinction-corrected magnitudes brighter than $b_{j}=19.45$. The main survey regions are two declination strips, one in the southern Galactic hemisphere spanning $80^{\circ} \times 15^{\circ}$ around the $\mathrm{SGP}$, and the other in the northern Galactic hemisphere spanning $75^{\circ} \times 10^{\circ}$ along the celestial equator; in addition, there are 99 fields spread over the southern Galactic cap. The survey covers $2000 \mathrm{deg}^{2}$ and has a median depth of $z=0.11$. A daptive tiling is used to give a highly uniform sampling rate of 93 per cent over the whole survey region. Redshifts are measured from spectra covering $3600-8000 \AA$ at a two-pixel resolution of $9.0 \AA$ and a median $S / N$ of 13 pixel $^{-1}$. A Il redshift identifications are visually checked and assigned a quality parameter $Q$ in the range 1-5; $Q \geq 3$ redshifts are 98.4 per cent reliable and have an rms uncertainty of $85 \mathrm{~km} \mathrm{~s}^{-1}$. The overall redshift completeness for $Q \geq 3$ redshifts is 91.8 per cent, but this varies with magnitude from 99 per cent for the brightest galaxies to 90 per cent for objects at the survey limit. The $2 \mathrm{dFGRS}$ data base is available on the World Wide Web at http://www. mso.anu.edu.au/2dFGRS.
\end{abstract}

Key words: surveys - galaxies: clusters: general - galaxies: distances and redshifts cosmology: observations - large-scale structure of U niverse. 


\section{INTRODUCTION}

The 2dF Galaxy Redshift Survey (2dFGRS) is designed to measure redshifts for approximately 250000 galaxies in order to achieve an order-of-magnitude improvement on previous redshift surveys, and provide a detailed and representative picture of the galaxy population and its large-scale structure in the nearby Universe (Colless 1999). The main goals of the $2 \mathrm{dFGRS}$ are as follows.

(1) To measure the galaxy power spectrum $P(k)$ on scales up to a few hundred $M p c$, filling the gap between the small scales where $P(k)$ is known from previous galaxy redshift surveys and the largest scales where $P(k)$ is well determined by observations of the cosmic microwave background (CM B) anisotropies. Particular goals are to determine the scale of the turnover in the power spectrum and to observe in the galaxy distribution the acoustic peaks detected in the CM B power spectrum (Percival et al. 2001).

(2) To measure the redshift-space distortion of the large-scale clustering that results from the peculiar velocity field produced by the mass distribution (Peacock et al. 2001). This distortion depends on both the mass density parameter $\Omega$ and the bias factor $b$ of the galaxy distribution with respect to the mass distribution, constraining the combination $\beta=\Omega^{0.6} / \mathrm{b}$.

(3) To measure higher order clustering statistics of the galaxy distribution in order to (a) determine the bias parameter $b$, revealing the relationship between the distributions of mass and light and yielding a direct measure of $\Omega$; (b) establish whether the galaxy distribution on large scales is a Gaussian random field, as predicted by most inflationary models of the early Universe, and (c) investigate the non-linear growth of clustering in the smallscale galaxy distribution.

(4) To fully and precisely characterize the galaxy population in terms of the distributions of fundamental properties such as luminosity, surface brightness, spectral type and star formation rate (Folkes et al. 1999; Cole et al. 2001; Cross et al. 2001; M adgwick et al. 2001a).

(5) To quantify the relationships between the internal properties of galaxies (such as luminosity, spectral type and star formation rate) and their external environment (the local density of galaxies and the surrounding large-scale structure) in order to constrain models of galaxy formation and evolution (N orberg et al. 2001).

(6) To investigate the properties of galaxy groups and clusters, not just from existing cluster catalogues (De Propris et al. 2001), but by defining a large, homogeneous sample of groups and clusters in redshift space, avoiding the problems of cluster catalogues defined in projection and allowing detailed study of the mass distributions and dynamical evolution in the most massive bound structures in the U niverse.

(7) To provide a massive spectroscopic data base for use in conjunction with other surveys, for finding rare and interesting types of object, and as a source for a wide variety of follow-up observations (Cole et al. 2001; M agliocchetti et al. 2001; Sadler et al. 2001).

This paper provides an overview of the survey and detailed description of the survey observations. The layout of the paper is as follows. Section 2 summarizes the main capabilities of the $2 \mathrm{dF}$ multifibre spectrograph; Section 3 describes the input source catalogue for the survey; Section 4 discusses the survey design, including the areas of sky covered by the survey and the tiling of the survey fields; Section 5 outlines the algorithm used to assign fibres to targets, and its uniformity and completeness; Section 6 describes the spectroscopic data obtained for the survey and the data reduction methods; Section 7 deals with the estimation of the redshifts and various internal and external checks on their reliability and precision; Section 8 describes the survey masks, which encapsulate the coverage, magnitude limits and redshift completeness of the survey; Section 9 outlines the main components and features of the survey data base; Section 10 summarizes some of the main results emerging from the survey, and Section 11 provides conclusions.

\section{THE 2DF SPECTROGRAPH}

The survey is designed around the Two-degree Field (2dF) multifibre spectrograph on the A nglo-A ustralian Telescope, which is capable of observing up to 400 objects simultaneously over a $2^{\circ}$ diameter field of view. Here we summarize the aspects of the instrument relevant to the $2 \mathrm{dFGRS}$; a full description is provided by Lewis et al. (2001) and the 2dF U ser M anual (http: / / www. aao.gov.au/2df). The four main components of $2 \mathrm{dF}$ are the corrector optics, the fibre positioner, the fibres and the spectrographs.

The $2 \mathrm{dF}$ corrector optics are a four-el ement lens assembly which gives 1-arcsec images over a 2:1 diameter flat field of view at the prime focus of the A AT. The corrector incorporates an atmospheric dispersion compensator (ADC), which corrects for atmospheric dispersion at zenith distances less than about $70^{\circ}$. The most significant remaining image degradation is a chromatic variation in distortion, which has the effect of dispersing images in the radial direction. This effect is largest halfway out from the field centre, where the wavelength range $0.35-1.0 \mu \mathrm{m}$ is dispersed over 2 arcsec radially. The radial distortion introduced by the corrector gives an image scale that varies over the field of view, from $15.5 \mathrm{arcsec} \mathrm{mm}^{-1}$ at the centre to $14.2 \mathrm{arcsec} \mathrm{mm}^{-1}$ at the edge; the corresponding change in focal ratio is from $f / 3.4$ to $f / 3.7$.

The $2 \mathrm{dF}$ fibre positioner $X-Y$ robot takes about $6-7 \mathrm{~s}$ on average to position one fibre. Since approximately 550-580 fibre moves are required to reconfigure a typical 400 -fibre field, this means that a full reconfiguration takes about 60-65 min. The actual configuration time varies, depending on the number of fibres used and the complexity of the field. To avoid dead-time there are two field plates, each with 400 fibres. While one field plate is placed at the focal plane for observing, the other is being reconfigured by the fibre positioner. The positions of the field plates are reversed by tumbling them about their horizontal axis.

The fibres are have $140-\mu \mathrm{m}$ diameter cores, corresponding to $2.16 \mathrm{arcsec}$ at the field centre and $1.99 \mathrm{arcsec}$ at the field edge. There are 400 fibres on each field plate. The internal precision with which the fibres are positioned is $11 \mu \mathrm{m}(0.16 \mathrm{arcsec})$ on average, with no fibres outside $20 \mu \mathrm{m}(0.3 \mathrm{arcsec})$. The fibres terminate in magnetic 'buttons' that attach to the steel field plates. Each button has a right-angle prism which directs the light from the focal plane into the fibre. To prevent fibre buttons coming into direct contact and to protect the fragile prisms, the fibres cannot be placed too close together. The absolute minimum fibre separation is $800 \mu \mathrm{m}$ (approximately 12 arcsec), and the required separation is generally larger (approximately $30 \mathrm{arcsec}$ ), as it depends on the detailed geometry of the buttons and their relative orientations.

Half the fibres on each field plate go to one of the two identical spectrographs. Each spectrograph has an $\mathrm{f} / 3.15$ off-axis M aksutov collimator feeding a $150-\mathrm{mm}$ collimated beam to the grating and then, at an angle of $40^{\circ}$, to an $\mathrm{f} / 1.2$ wide-field Schmidt camera. The detectors are Tektronix CCDs with $1024 \times 102424-\mu \mathrm{m}$ pixels. The 
200-fibre spectra imaged on to each CCD are separated by approximately 5 pixels. The $2 \mathrm{dFGRS}$ used the $300 \mathrm{~B}$ gratings, which are blazed at $4200 \AA$ and give a dispersion of $178.8 \AA \mathrm{mm}^{-1}$ $\left(4.3 \AA\right.$ pixel $\left.^{-1}\right)$. For a typical spectrograph focus of 2.1 pixels (FWHM), this corresponds to a FWHM spectral resolution of $9.0 \AA$. The $2 \mathrm{dF} G R S$ observations used a central wavelength around $5800 \AA$, and covered the approximate range $3600-8000 \AA$. Flexure is less than 0.2 pixel $\mathrm{hr}^{-1}$ (i.e., less than $40 \mathrm{~km} \mathrm{~s}^{-1}$ over a typical integration time).

The overall system efficiency (source to detector) of $2 \mathrm{dF}$ with the $300 \mathrm{~B}$ gratings used in the $2 \mathrm{dFGRS}$ is 2.8 per cent at $4400 \AA, 4.3$ per cent at $5500 \AA$ and 4.7 per cent at $7000 \AA$. These figures were obtained from measurements of photometric standard stars, corrected to nominal 1-arcsec seeing.

\section{SOURCE CATALOGUE}

The source catal ogue for the survey ( $M$ addox et al., in preparation) is a revised and extended version of the APM galaxy catalogue (Maddox et al. 1990a,b,c; Maddox, Efstathiou \& Suntherland 1996). This catalogue is based on A utomated Plate M easuring machine (APM) scans of 390 plates from the UK Schmidt Telescope (UKST) Southern Sky Survey. The extended version of the APM catalogue includes over 5 million galaxies down to $b_{j}=$ 20.5 in both north and south $\mathrm{G}$ alactic hemispheres over a region of almost $10^{4} \mathrm{deg}^{2}$ (bounded approximately by declination $\delta \leq+3^{\circ}$ and Galactic latitude $b \geq 30^{\circ}$ ). Small regions around bright stars, satellite trails and plate flaws are excluded; these are accounted for by the survey mask (see Section 8.1).

The $b$, magnitude system for the Southern Sky Survey is defined by the response of Kodak IIIal emulsion in combination with a GG395 filter. It is zero-pointed to Vega - i.e., bj is equal to J ohnson B for an object with zero colour in the Johnson-Cousins system. The colour equation is normally taken to be

$b_{j}=B-0.28(B-V)$,

following Blair \& Gilmore (1982). A larger coefficient $(-0.35)$ has been suggested by Metcalfe, Fong \& Shanks (1995), but we measure $-0.27 \pm 0.02$ in comparison with the ESO Imaging Survey (Arnouts et al. 2001), and we therefore retain the usual value of -0.28 . The photometry of the catal ogue is calibrated with numerous CCD sequences and, for galaxies with $b_{J}=17-19.45$, has a 68 per cent spread of approximately 0.15 mag, but with a nonGaussian tail to the error distribution. We emphasize that the calibration is to total CCD photometry, which absorbs any remaining correction to the thresholded A PM magnitudes.

The star-galaxy separation is as described in Maddox et al. (1990b), and the locus dividing stars and galaxies was chosen to exclude as few compact galaxies as possible, while keeping the contamination of the galaxy sample by stars to about 5 per cent. Spectroscopic identifications of the survey objects (see Section 7), show that the stellar contamination is in fact 6 per cent.

The source catalogue is incomplete at all magnitudes due to various effects, including the explicit exclusion of objects classified by the APM as merged images, the misclassification of some galaxies as stars, and the non-detection (or misclassification as noise) of some low surface brightness objects. This incompleteness has been studied in comparisons with deeper wide-area CCD photometry by Pimbblet et al. (2001) and Cross \& Driver (in preparation). The overall level of incompleteness is 10-15 per cent and varies slightly with apparent magnitude, being largest for the brightest and faintest objects. The main classes of objects that are excluded are (i) merged galaxy images that are explicitly excluded from the $2 \mathrm{dFGRS}$ source catalogue (about 60 per cent of the missing objects); (ii) large galaxies that are resolved into components that are classified as stellar, merged or noise objects (20 per cent); (iii) compact normal galaxies that are detected but classified as stars (15 per cent), and (iv) low surface brightness gal axies that are either not detected or classified as noise objects (5 per cent). Thus the main cause of incompleteness is misclassification of objects rather than their non-detection.

The target galaxies for the 2dFGRS were selected to have extinction-corrected magnitudes brighter than $b_{j}=19.45$. Since the targets were selected, improvements to the photometric calibrations and revised extinction corrections have resulted in slight variations to the magnitude limit over the survey regions this is precisely quantified by the magnitude limit mask for the survey (see Section 8.1). The $b_{j}$ extinction is taken to be $A_{b}=4.035 E(B-V)$, where the coefficient, and the reddening $E(B-V)$ as a function of position, come from Schlegel, Finkbeiner \& Davis (1998). The limit of $b_{\jmath}=19.45$ was chosen because (i) the surface density of galaxies at $b_{j}=19.45$ (approximately $165 \mathrm{deg}^{-2}$ ) is sufficiently larger than the surface density of $2 \mathrm{dF}$ fibres on the sky $\left(127 \mathrm{deg}^{-2}\right)$ to allow efficient use of all fibres - few fibres are unused even in low-density fields, and (ii) the time taken to configure a typical field (60-65 min) allows, with overheads, a sufficiently long exposure time to reach the desired signal-to-noise level of $S / N>10$ pixel $^{-1}$ for galaxies with $b_{j}=19.45$ even in rather poor conditions. This limiting magnitude corresponds to a median redshift for the survey of about $z=0.1$, so that the $2 \mathrm{dFGRS}$ is essentially a survey of the local Universe.

\section{SURVEY DESIGN}

\subsection{Survey areas}

The areas of the sky covered by the survey were chosen so as to satisfy a number of different requirements. The first goal was to cover as large a volume as possible, in order to closely approach a statistically representative sample of the Universe on the largest possible scales. The second was to obtain near-complete sampling down to the survey limit in order to have the finest possible resolution of structure on small scales. The third requirement was to match the sample to the observational capabilities of the $2 \mathrm{dF}$ instrument in order to achieve high efficiency. The adopted geometry is an effective compromise between these requirements.

The survey consists of two separate declination strips of overlapping $2^{\circ}$ fields plus 99 scattered 'random' $2^{\circ}$ fields. One strip (the SGP strip) is in the southern Galactic hemisphere and covers approximately $80^{\circ} \times 15^{\circ}$ centred close to the South Galactic Pole $\left(21^{\mathrm{h}} 40^{\mathrm{m}}<\alpha<03^{\mathrm{h}} 40^{\mathrm{m}},-37^{\circ} .5<\delta<-22^{\circ} .5\right)$. The other strip (the NGP strip) is in the northern Galactic hemisphere and covers $75^{\circ} \times 10^{\circ}\left(09^{\mathrm{h}} 50^{\mathrm{m}}<\alpha<14^{\mathrm{h}} 50^{\mathrm{m}},-7.5<\delta<+2.5\right)$. The 99 'random' fields are chosen from the low-extinction region of the APM catalogue in the southern Galactic hemisphere outside the survey strip (the mean extinction over each field is required to be less than 0.2 mag - see fig. 2 of Efstathiou \& M oody 2001). The fields are chosen pseudo-randomly within this region, except that the field centres are at least $3^{\circ}$ apart. A map of the survey fields on the sky is shown in Fig. 1; the locations of the fields with respect to the extinction map derived from Schlegel et al. (1998) are shown in Fig. 2. All the survey fields lie at Galactic latitudes greater than 


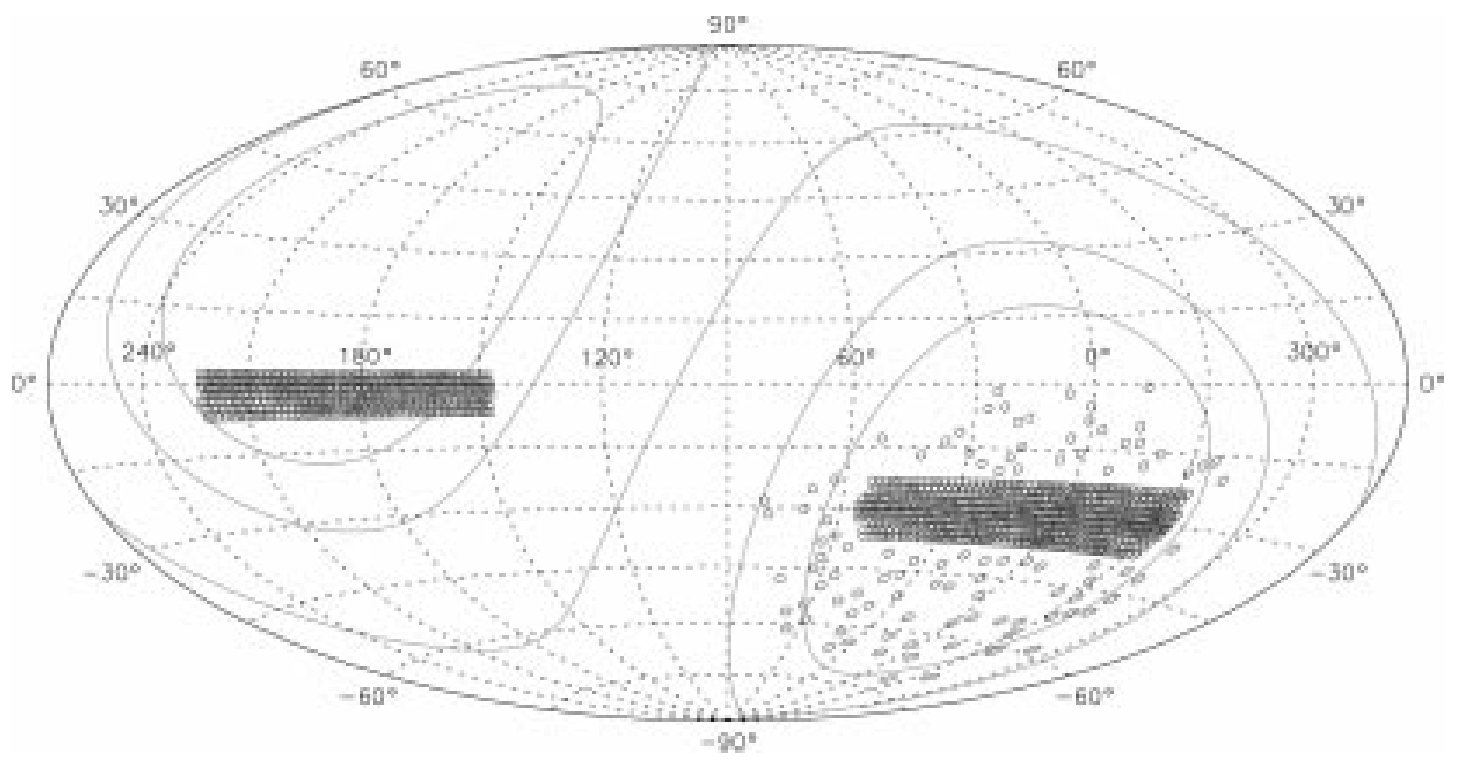

Figure 1. The $2 \mathrm{dFGRS}$ regions shown in an Aitoff projection of RA and Dec., with individual $2 \mathrm{dF}$ fields marked as small circles. A lso shown are the lines of Galactic latitude $|\mathrm{b}|=0^{\circ}, 30^{\circ}$ and $45^{\circ}$. The numbers of survey galaxies in these regions are 193550 in the 643 fields of the $80^{\circ} \times 15^{\circ} \mathrm{SGP}$ strip, 139144 in the 450 fields of the $75^{\circ} \times 10^{\circ} \mathrm{NGP}$ strip, and 57019 in the 99 fields scattered around the SGP strip.
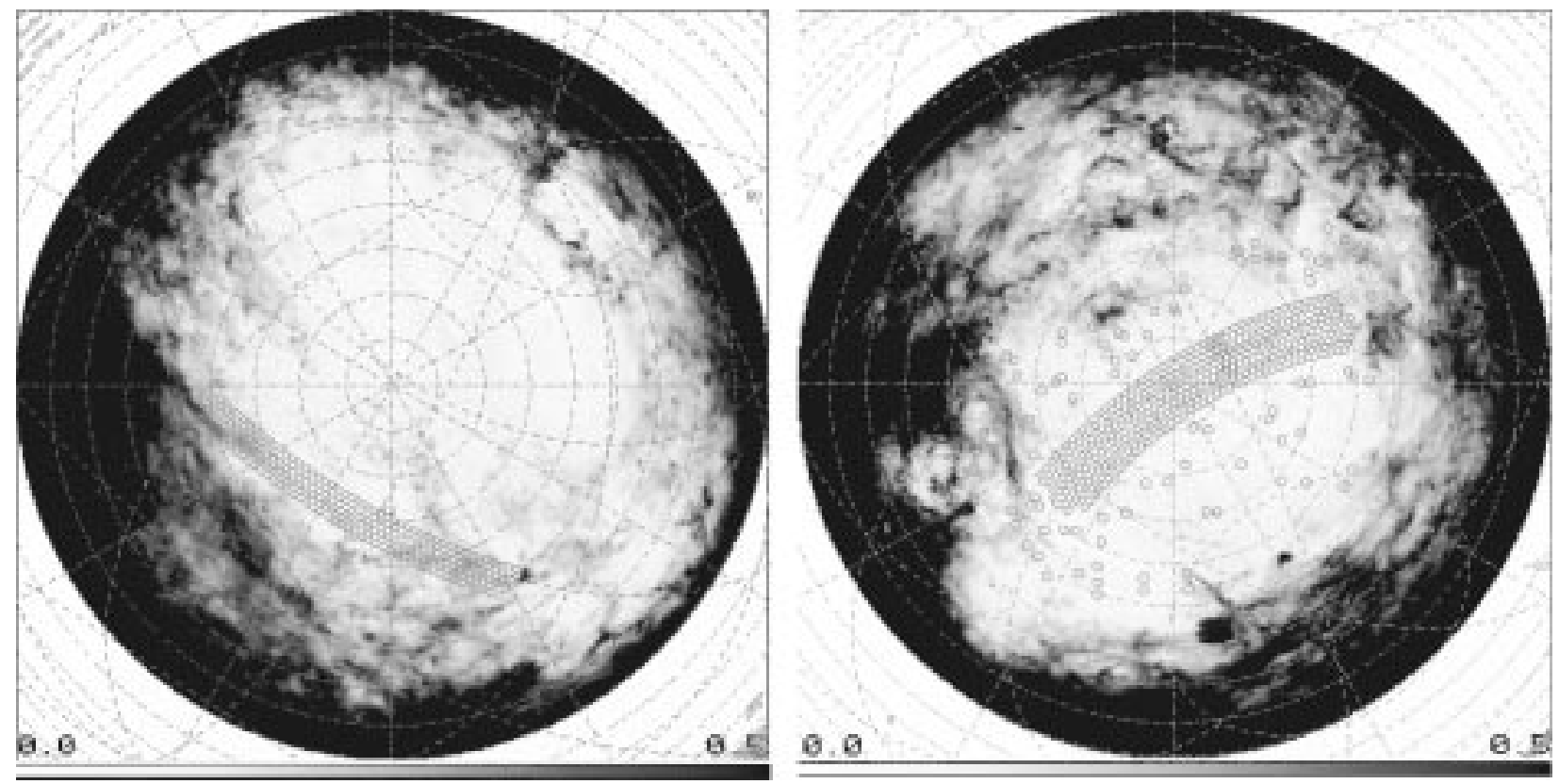

Figure 2. The survey fields in the NGP (left) and SGP (right) on maps of the extinction $A_{b_{1}}$ derived from Schlegel et al. (1998).

$|b|=30^{\circ}$, and the whole of the SGP strip, and most of the NGP strip and the random fields, lie at Galactic latitudes greater than $|b|=45^{\circ}$.

The distribution of extinction corrections as a function of Galactic latitude, and the fraction of corrections larger than a given value, are shown in Fig. 3. Overall, the median correction is 0.07 mag, 90 per cent are less than 0.16 mag, and 99 per cent are less than $0.26 \mathrm{mag}$; the corresponding quantiles in the NGP are $(0.12,0.19,0.28) \mathrm{mag}$, in the SGP $(0.05,0.07,0.11) \mathrm{mag}$, and in the random fields $(0.07,0.13,0.30)$ mag.

The 2dFGRS target sample of galaxies contains 193550 galaxies in the SGP strip, 139144 galaxies in the NGP strip, and 57019 galaxies in the random fields. This gives a total of 389713 possible targets, significantly more than the survey goal of 250000 galaxies. Survey observations of the NGP and SGP strips are proceeding outwards in declination from the centre of each strip towards this goal. $\mathrm{N}$ ote that the total number of galaxies listed in the survey source catalogue (and the survey data base) is 467214 , which is larger than the number of possible survey targets because the source catalogues for the NGP and SGP strips conservatively include galaxies fainter than the spectroscopic survey magnitude limit, down to $b_{\jmath}=19.6$.

At the median redshift of the survey $(z=0.11)$ the SGP strip extends over $400 \mathrm{~h}^{-1} \mathrm{Mpc} \times 75 \mathrm{~h}^{-1} \mathrm{Mpc}$, and the NGP strip over $375 \mathrm{~h}^{-1} \mathrm{M} \mathrm{pc} \times 50 \mathrm{~h}^{-1} \mathrm{M} \mathrm{pc}$. Out to the effective limit of the survey at $z \approx 0.3$, the strips contain a volume of $1.2 \times 10^{8} \mathrm{~h}^{-3} \mathrm{M} \mathrm{pc}^{3}$ (for $\Omega_{\mathrm{m}}=0.3, \Omega_{\Lambda}=0.7$ ); the volume sparsely sampled including the random fields is between 2 and 3 times larger. 


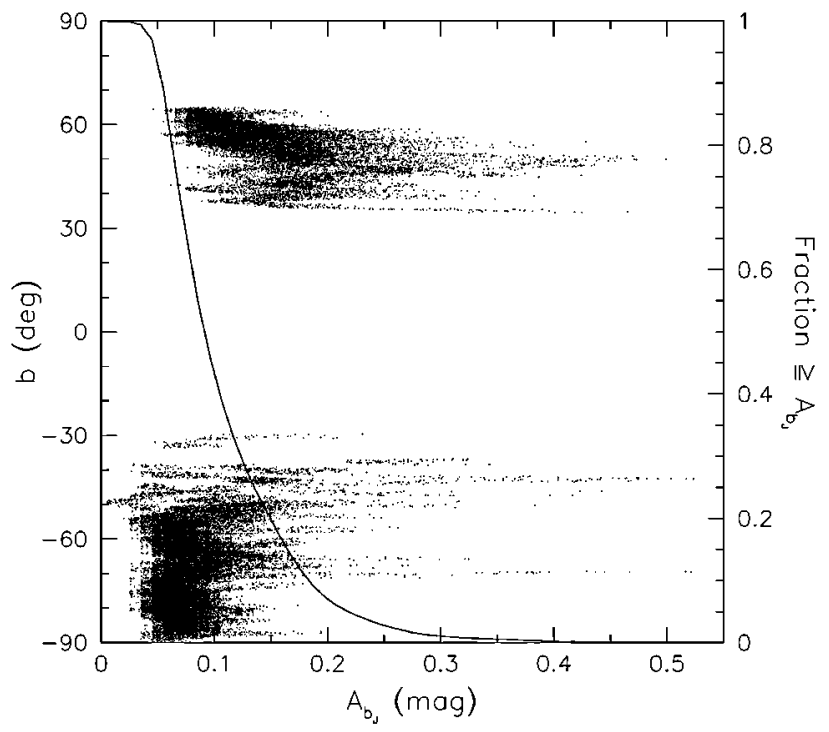

Figure 3. The distribution of extinction corrections $A_{b}$, with Galactic latitude $b$ (dots and left axis), and the fraction of corrections larger than $a$ given value (line and right axis).

\subsection{Tiling the survey}

The survey limit of $b_{j}=19.45$ was chosen, in part, because it gives a good match between the surface density of galaxies and the surface density of $2 \mathrm{dF}$ fibres. Due to clustering, however, the number of galaxies in a given field varies considerably. The rms variation in the number of galaxies per randomly placed $2^{\circ}$ field is 140 at $b_{j}=19.5$, and is largely independent of the choice of magnitude limit over the range considered here. To make efficient use of $2 \mathrm{dF}$, we therefore require an algorithm for tiling the sky with $2^{\circ}$ fields that allows us to cover the survey area at a high, and nearly uniform, sampling rate with the minimum number of $2 \mathrm{dF}$ fields.

So long as the sampling of the source catal ogue is not biased in any way that depends on the photometric or spectroscopic properties of the galaxies, we can always use the source catalogue to accurately determine the sampling rate as a function of position (see Section 8). The sampling can then be accounted for in any analysis. However, to keep such corrections to a minimum, considerable effort has been invested in making the sampling as complete and uniform as possible.

There are a number of possible approaches to laying down target field centres. The simplest is to adopt a uniform grid of equally spaced centres, and then either randomly sample each field with the number of available fibres or observe each field several times until all the galaxies have been observed. The second of these options is clearly inefficient, as it will give rise to a large number of fields being observed with significantly less than the full complement of fibres, while the first is undesirable as it gives a different sampling factor for each field. A more efficient solution is to use an adaptive tiling strategy, where we allow each field centre to drift from the regular grid so that we maximize the number of targets that are assigned to each field, subject to the constraint that the number of targets assigned to any one field should not exceed the number of available fibres, $\mathrm{N}_{\mathrm{f}}$.

We begin with a uniform grid with field centres equally spaced by $s=\sqrt{3^{\circ}}$ in right ascension along rows 1.5 apart in declination. For each galaxy in the survey we then determine how many fields it lies within, and assign each field a weight $w_{i}$, where

$$
\begin{aligned}
\mathrm{W}_{\mathrm{i}} & =0 & & \text { if } \mathrm{N}_{\mathrm{i}} \geq \mathrm{N}_{\mathrm{f}} \\
& =1-\frac{\mathrm{N}_{\mathrm{i}}}{\mathrm{N}_{\mathrm{f}}} & \text { if } & \mathrm{N}_{\mathrm{i}}<N_{\mathrm{f}}
\end{aligned}
$$

and $\mathrm{N}_{\mathrm{i}}$ is the number of galaxies in this field. These weights are normalized such that $\sum_{1}^{N_{t}} W_{i}=1$, where $N_{t}$ is the number of fields that this galaxy could be assigned to. The galaxy in question is then randomly assigned to one of the fields using these weights, unless all the fields are already filled, in which case it is assigned to the first field in which it was found. Once all the field occupancies have been determined in this way, we move each field in right ascension by an amount

$$
\begin{aligned}
\delta \alpha_{\mathrm{i}} & =\Delta \alpha+0.05 \mathrm{~s} \frac{\mathrm{N}_{\mathrm{i}}}{\mathrm{N}_{\mathrm{f}}} \quad \text { if }\left(\mathrm{N}_{\mathrm{i}} \geq \mathrm{N}_{\mathrm{f}}\right) \\
& =\Delta \alpha-\mathrm{s}^{\prime}\left(1-\frac{\mathrm{N}_{\mathrm{i}}}{\mathrm{N}_{\mathrm{f}}}\right) \quad \text { if }\left(\mathrm{N}_{\mathrm{i}}<\mathrm{N}_{\mathrm{f}}\right),
\end{aligned}
$$

where $s$ is the maximum allowed separation between adjacent fields, $s^{\prime}$ is the current distance to the neighbouring field centre, and $\Delta \alpha$ is the cumulative shift that has currently been applied to this row. New fields are added at the end of each row if the total length of the row has contracted enough to exclude any galaxies at the trailing edge. We found that using a fixed separation of 1.5 in declination, and adjusting the tile positions in right ascension only, provided sufficient flexibility to achieve uniform high completeness without a large increase in the total number of fields and without leaving gaps in the sky coverage.

In practice, it is found that the above prescription requires a further modification to account for the position of each object within the field. This additional constraint arises because of the physical restrictions on the positioning of individual fibres, both in terms of their deviation from the radial angle and their extension from the parked position at the edge of the field. We apply this constraint by dividing each field into 36 subfields and restricting the number of targets that can be assigned to each subfield to 16 . This is larger than the number of fibres whose park positions fall within the arc of the sector, but allows for the fact that the area of the field that can be reached by each fibre is increasing with the fibre extension. W ithout this extra constraint the al gorithm tends to place large clusters in the overlapping areas of neighbouring fields where, although there are more available fibres because of the overlap, it rapidly becomes impossible to use these fibres because of the high density of targets close to the edge of each field. Limiting the number of targets within each sector effectively removes this problem, and so increases the uniformity of the survey completeness.

The adaptive tiling algorithm also needed to cope with the requirement that the galaxy redshift survey be merged with the concurrent survey of QSO candidates (the 2dF QSO Redshift Survey: Boyle et al. 2000; Croom et al. 2001). This results in a higher surface density of targets in the region of overlap, for which we compensate by reducing the separation in declination of the tiling strips in the QSO survey regions to 75 per cent of the original value (i.e., to 1.125). As the QSO survey occupies the central declination strip of our SGP survey region this results in a 3-4-3 arrangement of tiling strips over the three rows of UKST fields used in the SGP survey. A similar consideration applied to the NG P survey region gives a 3-4 arrangement if we consider the full areas of the two rows of UKST fields used.

With these additional modifications, our tiling algorithm was 
able to achieve an overall sampling rate of 99.8 per cent, dropping to 93 per cent after the assignment of fibres to targets has been made (see Section 5.3). On average, fewer than 5 per cent of the fibres in each field were unused. For comparison, the mean sampling rate that would be achieved for randomly distributed fields is 68 per cent, with an rms variation of 15 per cent.

\section{FIBRE ASSIGNMENT}

The tiling procedure described above fixes the location of the $2 \mathrm{dF}$ target fields on the sky and provides a first-pass priority scheme (based on the random field assignments) for use in assigning targets to fibres. How ever, the mechanical constraints of $2 \mathrm{dF}$ imply that we cannot usually allocate fibres to all the targets assigned to a particular field, and so we consider all targets that lie within each field boundary. Our tiling scheme implies that many of the survey targets are found on more than one field, so we adopt a priority scheme as follows: targets which are unique to the field in question are assigned the highest priority, then targets which are assigned to the field, but which can also be reached from neighbouring fields, then targets which fall within the field, but which are assigned to a different, overlapping field. The priorities of the QSO targets are increased one step to ensure that we do not imprint the strong clustering pattern of the galaxy distribution on the weak clustering expected from the QSO sample.

\subsection{Single-field assignments}

The allocation procedure for a single field can be divided into two distinct steps: an initial allocation pass and a fibre-swapping pass. These two steps are repeated for each range of decreasing priorities for targets that have been assigned to this field. A $n$ attempt is then made to reduce the complexity of the configuration by identifying pairs of allocated fibres which are crossed, and which can safely be exchanged. The allocation and uncrossing steps are then repeated to allow consideration of the lower priority targets which were not assigned to this field. The details of these steps are as follows.

(1) Initial allocation pass. For each target, t, of a given priority we determine the set, $\mathcal{F}_{\mathrm{t}}$, of all fibres that could be allocated to that target in the absence of any other allocations. We then determine the set, $\mathcal{T}_{\mathrm{t}}$, of all targets which fall within the sector defined by the target and the two most extreme fibres in $\mathcal{F}_{\text {t. }}$. We then use the difference $\mathrm{N}\left(\mathcal{T}_{\mathrm{t}}\right)-\mathrm{N}\left(\mathcal{F}_{\mathrm{t}}\right)$ between the number of targets in $\mathcal{T}_{\mathrm{t}}$ and the number of fibres in $\mathcal{F}_{t}$ to determine which targets are hardest to access and hence should be given the highest priority: targets within the sector defined by the target with the largest value of $\mathrm{N}\left(\mathcal{T}_{\mathrm{t}}\right)-\mathrm{N}\left(\mathcal{F}_{\mathrm{t}}\right)$ are allocated first. This procedure is then repeated for targets of successively lower priorities.

(2) Fibre-swapping pass. For each remaining unallocated target, $u$, we now consider each fibre in $\mathcal{T}_{u}$. For each fibre in this set that could still be allocated to $u$ given the current configuration we make a tentative re-allocation of this fibre from its initial assignment, i, to target $u$, and check to see if any unallocated fibres can be assigned, to i. If no unallocated fibres can be assigned we repeat this process recursively until one of the following conditions have been satisfied: either (i) a previously unallocated fibre is allocated (implying that $u$ has been allocated and that all previously allocated targets remain allocated), or (ii) the search exceeds a depth of 10 iterations. In the latter case, if at any point in the search a fibre has been moved from a low-priority target such that a target of higher priority has been allocated, then the search is unpacked to this point; if no such trade-off was found, then the search is unpacked to the original configuration.

(3) Fibre-uncrossing pass. Each pair of fibres in the configuration is tested to see if the fibres cross. For each crossed pair an attempt is made to swap the allocations of fibres to targets. This process is iterated until no further swaps can be made. The net effect of this procedure is that the final configuration supplied to the positioner is simplified so that the transition to the following configuration will require fewer fibres to be moved twice. This simplification effectively reduces the time required for each reconfiguration, and is achieved without constraint on the allocation.

\subsection{Field-overlap assignments}

While the above procedure provides an optimized method of allocating as many fibres as possible to a given individual field, it does not make optimal use of the flexibility provided by the overlapping fields. We therefore ran a second pass of the configuration algorithm which considered each pair of overlapping fields as an 800-fibre problem in which all objects found within the overlap area are matched up, so that an object which is allocated in one field can be allocated to the second field to allow acquisition of unallocated targets in the first field. Compared to the results of treating the two overlapping fields as separate configuration problems, this procedure proves more effective at reducing the overall incompleteness due to the instrumental constraints. Since the tiling algorithm tends to place local over-densities close to the edges of tiles, this procedure also serves to homogenize the distribution of incompleteness as a function of position within a field.

A significant fraction of this incompleteness arises due to close pairs of objects which lie within the unique regions of individual fields. The separation at which this occurs is a strong function of the relative orientation of the two fibre buttons (see Lewis et al. 2001). The upper limit on the value of this separation is $11 \mathrm{~mm}$ in the focal plane (corresponding to $2.2 \mathrm{arcmin}$ ), while the mean separation of close pairs of objects for which one target cannot be assigned is $\sim 30$ arcsec.

\subsection{Fibre assignment completeness}

The results of this procedure are that we are able to allocate fibres to 93 per cent of the source catalogue objects. The distribution of the unallocated objects on the sky are shown in Fig. 4. The most prominent features visible in these distributions are occasional localized clusters of unallocated objects. These are due to overdense regions where the geometrical packing constraints imposed by the fibre button dimensions mean that it has not been possible to assign every fibre to a target, even though there are enough fibres available as a result of the tiling algorithm. This effect is enhanced by the relative increase in the number of close pairs in strongly clustered regions.

The distribution of these unallocated objects is also shown as a function of position within the $2 \mathrm{dF}$ field in Fig. 5. This plot shows a slight overall gradient in the RA direction, which is due to the order in which the field overlaps are processed (see Section 5.2). This apparent gradient is misleading, since the missed objects are plotted according to the field plate coordinates on the plate to which they were assigned, whereas the majority of objects which contribute to this effect are actually missed on more than one $2 \mathrm{dF}$ field. The next most prominent feature of Fig. 5 is the imprint of the 

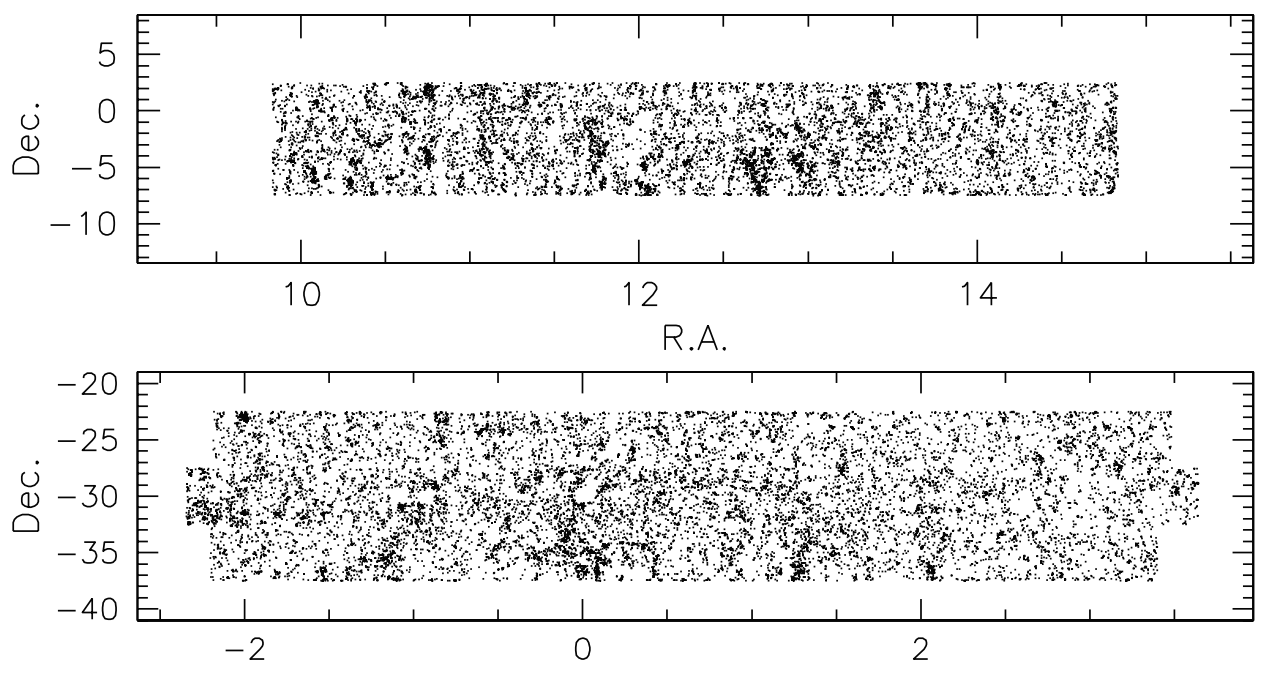

R.A.

Figure 4. The distribution of the 7 per cent of objects in the sample that are not allocated to fibres (NGP strip at top, SGP strip at bottom).

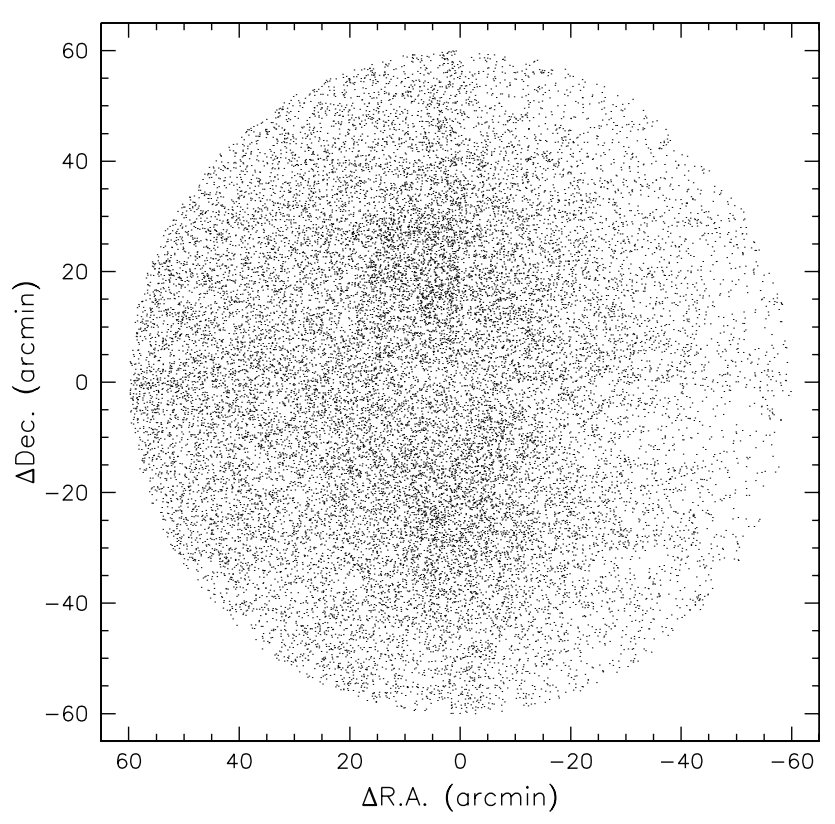

Figure 5. The distribution of the unallocated objects within the stacked $2 \mathrm{dF}$ fields.

four fibres which are used for field acquisition and guiding. These fibres must be allocated with a higher priority than the targets if the field is to be observed successfully. They prevent the subsequent allocation of target objects lying al ong their length, and so produce a slight increase in the numbers of unallocated objects along the four cardinal axes of the field, in the regions of the plate where they can be placed.

It should be noted that these figures do not represent the final incompleteness in the survey, since individual target allocations are adjusted immediately prior to observation to account for the actual number of fibres available to $2 \mathrm{dF}$ on any given night (typically, about 1 per cent of the fibres are broken at any given time, al though they are continually replaced). The overall incompleteness at this stage, before any observations have been made, is 7 per cent. The level of this incompleteness is such that the pattern is dominated by residuals due to clustering within individual $2^{\circ}$ fields, implying that an attempt to correct for this effect would effectively imprint the observed pattern on to the data. We therefore conclude that we have achieved our goal of reducing the mean incompleteness to an acceptably low level, comparable to the level of incompleteness due to the individual uncertainties in galaxy magnitudes and the incompleteness due to not measuring redshifts because of inadequate signal-to-noise ratio $(\mathrm{S} / \mathrm{N})$. We emphasize that neither the tiling nor the fibre allocation depends on the photometric or spectroscopic properties of the objects, so that we can use the source catalogue to accurately determine the sampling of the survey, as discussed in Section 8.

\subsection{Visual inspection}

Postage stamp images of each target galaxy and each candidate fiducial star were generated from the Digitized Sky Survey ${ }^{1}$ (DSS) (Lasker et al. 1998). These DSS images were used to examine the target galaxies for merged images and to remove unsuitable fiducial star candidates.

The APM image classification parameter $k$ (see Maddox et al. 1990b) was used to find target galaxies which might possibly be merged images. Objects with $k>1.15$ in the SGP and $k>1.2$ in the NGP were analysed by a simple routine which attempts to relocate the fibre on to the local maximum of surface brightness closest to the nominal target position. This is achieved by starting from the central pixel of the DSS image and allowing the position to move in a direction of increasing intensity until a local maximum is found. The position corresponding to this local maximum is then inserted into the $2 \mathrm{dF}$ configuration file prior to observation. A ll objects which are tested in this way are flagged as merged images by changing the second character of the object's OBSNAME parameter from $\mathrm{G}$ to $\mathrm{M}$, even if the final position of the fibre is unchanged. The survey data base records both the original source catalogue position (data base parameters RA and DEC; see Section 9.2) and the actual observed position (data base parameters OBSRA and OBSDEC); the parameter MATCH_DR gives the offset

\footnotetext{
${ }^{1}$ The Digitized Sky Survey was produced at the Space Telescope Science Institute, and is based on photographic data obtained using the Oschin Schmidt Telescope on Palomar M ountain and the UK Schmidt Telescope.
} 
(in arcsec) between these two positions, and may be non-zero for objects flagged as mergers.

The DSS images of candidate fiducial stars are inspected visually to allow removal of the following types of object, which would compromise field acquisition: (i) stars which are merged with another object; (ii) stars which are close to other stars of comparable brightness; (iii) bright stars which are strongly saturated on the sky survey plates; (iv) spurious objects that are fragments of the diffraction spikes or hal os of bright stars, or bright regions within large galaxies; ( $v$ ) asteroids and portions of satellite trails, and (vi) spurious objects due to noise.

\subsection{Final adjustments}

The configuration procedure described above determines the optimal assignment of fibres to targets over the whole survey. This procedure assumes a fixed set of parameters for the transformation of sky-coordinates to plate-coordinates, whereas in practice a slightly different transform is determined each time the instrument is mounted on the telescope. The above procedure also assumes a full complement of fibres, and does not allow for the small but significant attrition rate as fibres are disabled due to optical or mechanical failures. Fibres which are disabled in this way are repaired on a regular basis, but operational constraints require that disabled fibres are replaced in batches, and so there is generally some delay before a particular fibre is replaced.

We account for both the fibre attrition and the effects of changes to the coordinate transform by re-examining each configuration the day before that field is to be observed. A II broken or inoperative fibres which had been allocated are deallocated, and the targets to which they were assigned are flagged. A $n$ attempt is then made to recover each flagged target using the current list of available fibres, according to the same algorithm used for the original allocation. A gain, low-priority targets may be lost at this stage in favour of high-priority targets. In practice, we usually find that the majority of the high-priority targets which were flagged can either be recovered or replaced.

A similar check is then made to allow for a further change to the coordinate transform due to the hour angle at which the field is to be observed. This correction is a combination of the effects of atmospheric refraction and flexure and misalignment of the telescope itself. Each configuration is checked for hour angles of $\pm 4 \mathrm{~h}$, and adjustments are made to ensure that no fibre conflicts will occur within this range. Again, we usually find that all such conflicts can be recovered without loss of target allocations.

After this correction to the configuration, a number of unallocated fibres (at least 10 for each spectrograph) are allocated to blank sky positions. This is done initially using an azimuthally symmetrical grid of positions around the field, but if insufficient fibres can be allocated to this grid, more are allocated to other blank sky regions manually. The position of each allocated sky position is then checked to ensure that it is genuinely blank by examining the surrounding area using the DSS. A ny sky fibres likely to be contaminated by objects visible on the DSS are reallocated to other blank sky regions.

\section{SPECTROSCOPIC DATA}

\subsection{Observational procedure}

A target field is acquired using the four guide fibres, each of which consists of six fibres in a hexagonal pattern surrounding a central fibre. The individual fibres are $95 \mu \mathrm{m}$ (1.4 arcsec) in diameter and have centre-to-centre separations of $1.8 \mathrm{arcsec}$. These guide fibres are positioned on $14<\mathrm{V}<15$ stars with positions measured from the same U K ST plates as the target galaxies. A s the blue sky survey plates are often 20-25 years old, we use the more recent red sky survey plates to check for any proper motion, and we also apply a colour cut to reduce the number of nearby stars. On some fields, where red plates are not available, we have used fiducial stars from the USNO A 1.0 astrometric catalogue (M onet et al. 1996).

The guide fibres are viewed by the AAT's standard acquisition and guiding TV system. The position for optimum acquisition of the fiducial stars, and subsequent guiding corrections, are determined by visual inspection of the locations of the stars in the guide fibres on the TV image. Acquisition of a new field typically requires less than $5 \mathrm{~min}$.

For each field we first take a multifibre flat-field exposure using the quartz lamp in the calibration unit. This flat-field is used to trace the positions of the fibres on the image, to fit the spatial profile of each fibre as a function of wavelength, and to apply a one-dimensional pixel-to-pixel flat-field correction to the extracted spectra. We next take a wavelength calibration exposure of helium and copper-argon arc lamps. We then typically take three 1100-s exposures of the target objects. However, the exposure time is varied to suit the conditions: for galaxy-only fields in good conditions, three 900 -s exposures are sufficient; in poor conditions, especially for fields including targets from the QSO survey, a longer total integration time is used. With the CCD readout time of $60 \mathrm{~s}$, the whole series of exposures typically takes just over an hour, so that the observations of one field are finished just as the configuration for the next field is completed.

\subsection{Data reduction}

The data are reduced using the $2 \mathrm{dF}$ data reduction pipeline software, $2 \mathrm{dfdr}$, a full description of which is given by Bailey et al. (2001; see also http://www. aao.gov.au/2df). The main steps in the process are as follows.

(1) Basic image processing. Each image undergoes the same initial processing, which consists of flagging bad pixels (both saturated pixels and those specified in the bad-pixel mask for each CCD). The bias level is computed as the median of the CCD overscan region and subtracted from the image. The image is then trimmed to remove the overscan region. Finally, a variance array is computed based on the pixel data values and the known properties of the CCDs. The variances are propagated through all subsequent reduction steps.

(2) Mapping the spectra. The next step is to map the locations and shapes of the spectra on the detector. The centroids of the fibre spectra al ong the slit are determined using the fibre flat-field image, and the fibre identifications are checked visually. This is necessary because the positioning of the slit block is not precisely reproducible, so that the locations of the fibres on the CCD can change by a few pixels, and occasionally the first or last fibre may fall off the edge of the detector. Visual checking ensures that spectra are correctly identified with fibres and thus with the objects that were observed. Given this starting location, $2 \mathrm{dfdr}$ finds the centroids of each fibre spectrum as a function of wavelength and fits a model based on the known optical properties of the spectrograph. For each subsequent frame, this model is adjusted (to account for flexure) by fitting an overall shift and rotation to the positions of the spectra on the detector. 
(3) Subtracting the background. The background of scattered light is fitted with an empirically determined model using the unilluminated portions of the detector. Fibres are so closely packed on the detector that the light level between transmitting fibres does not drop to the background level. Hence the only unilluminated areas are at the edges of the detector and at the positions of dead (broken, non-transmitting) fibres. A small number of fibres are broken at any one time, and are continually being replaced. Occasionally the number of broken fibres becomes sufficiently small that it is not possible to reliably fit the background, and this step must be omitted.

(4) Fitting the spatial profiles. The close packing of the fibres on the detector means that their spatial profiles overlap, producing cross-talk between neighbouring spectra. In order to correct for this, it is necessary to know the spatial profile of each fibre spectrum as a function of wavelength. These profiles are determined from the fibre flat-field image by performing simultaneous fits of 200 Gaussians to the fibre profiles at each of 20 wavelength ranges. These fits are then interpolated in the wavelength direction with cubic splines to give the spatial profile for each fibre at every wavelength on the detector.

(5) Spectrum extraction. Given the location and shape of each fibre's spatial profile, optimal extraction consists of a weighted fit of the profiles to recover the amplitude of the spectrum at each wavelength. B ecause the spectra overlap, a simultaneous fit of the profile amplitudes of each fibre spectrum and its nearest neighbour on either side is carried out. The fit is performed using leastsquares with variance weighting.

(6) F lat-fielding. The extracted spectra are then 'flat-fielded' by dividing by the pixel-to-pixel variations in the extracted spectra from the fibre flat-field image. This one-dimensional flat-fielding is used because there is presently no means of generating a full twodimensional flat-field image.

(7) Wavelength calibration and linearization. A predicted wavelength for each pixel, based on the nominal central wavelength and the optical model for the spectrograph, is first calculated. Lines in the helium/argon arc spectra are automatically identified by a peak-finding algorithm, then matched to a list of known lines, discarding both unidentified peaks and unmatched lines, as well as known blends. The relation between the measured line positions and their true wavelengths is fitted by a third-order polynomial. This fit iterated up to four times, with the most discrepant line excluded at each iteration. The final fits usually include 21-22 lines over the $4400-\AA$ spectral range, and have typical rms residuals of $0.3 \AA$ ( 0.07 pixels). Once the wavelength calibration is determined, the spectrum is rebinned on to a linear wavelength scale using quadratic interpolation.

(8) Fibre throughput calibration. The spectrum of the sky background is measured with at least eight fibres (and usually more) on each of the two spectrographs. The spectra from the sky fibres are normalized by their mean fluxes and a median sky spectrum is calculated. The relative throughputs of the fibres are then derived from the relative fluxes in the strong sky lines as follows. All object and sky fibres and the median sky are continuum-subtracted using a continuum derived by median smoothing with a 201-pixel box. This removes the continuum, leaving only the strong lines in the residual spectrum. A robust least-squares fit to the constant of proportionality between the counts in each pixel of the residual object+sky spectrum and the residual median sky spectrum then yields the relative throughput for each fibre (the sky lines in common to both spectra contribute to the fit, but strong emission lines found only in the object spectrum do not). The individual object and sky spectra are then normalized by dividing by their relative throughputs.

(9) Sky subtraction. The median sky spectrum is recalculated from the individual normalized sky spectra, and subtracted from each normalized object and sky spectrum. The sky-subtraction precision, measured as the ratio of the total flux in the sky fibres before and after sky-subtraction, is typically 2-3 per cent and rarely worse than 6 per cent. Precise sky-subtraction is dependent on effective scattered light subtraction, wavelength calibration and throughput calibration.

(10) Combining spectra. The fully reduced spectra from each of the multiple exposures on the target objects (typically three) are optimally combined in a final step. The combination algorithm accounts for overall flux variations between exposures (due to different integration times or observing conditions) and rejects cosmic ray events. The relative weighting of the exposures is computed from the total fluxes in the brightest 5 per cent of the spectra (after they are median-smoothed over a 201-pixel box to eliminate cosmic rays and normalized to the first exposure). The maximum flux weight is unity, and the weights are applied to the individual exposures before they compared for cosmic rays. The cosmic ray rejection algorithm is similar to that of the IRAF crreject package. It flags likely cosmic ray events, which are taken to be those pixels that are more than $5 \sigma$ deviations from the median (over the set of flux-weighted exposures) together with some neighbouring pixels. The final pixel value in the combined spectrum is computed as the variance-weighted mean over the fluxweighted exposures of the unflagged pixels.

The final result of the reduction is a pair of data files (one for each CCD), both of which contain a multispectrum image (200 fibres $\times 1024$ spectral pixels), the associated variance array (which is correctly propagated throughout the reduction process), and a copy of the median sky spectrum used in the background subtraction. The data files also contain the identifications of the spectra obtained from the fibre configuration file and detailed information about the instrument and observation.

The spectra are not flux-calibrated. Flux calibration of fibre spectra is very difficult to achieve for a number of reasons. Chief among these are (i) astrometric/positioning errors, which, combined with the small fibre aperture, prevent reproducible sampling of each galaxy's light distribution, and (ii) chromatic variations in distortion (see Section 2) and residual errors in the atmospheric dispersion correction, which produce variations in the flux calibration dependent on the attitude of the telescope and the position of the object in the field. These effects can produce significant variations in the continuum slope of the spectra, and are discussed in quantitative detail in M adgwick et al. (2001b), and in preparation).

Various problems are exhibited by some of the spectra. (i) Poor sky-subtraction. A small but significant fraction of fields suffered sky-subtraction errors of more than 5 per cent. The strong oxygen sky lines at 5577,6300 and $6363 \AA$, and the $\mathrm{OH}$ bands to the red of $6000 \AA$, leave significant residuals in the spectra. (ii) Fringing. Damaged fibres sometimes produce a fringing effect that results in a strong oscillation in the throughput with wavelength. (iii) Halation. Sometimes halation due to condensation on the field flattener lens just in front of the CCD results in a high level of scattered light which fills in absorption features; when present, this effect was worse in $C C D$ camera \#2. (iv) ADC error. A software error meant that the atmospheric dispersion corrector (ADC) was incorrectly positioned for observations prior to 1999 
A ugust 31; some of the spectra obtained before this date suffer from significant atmospheric dispersion losses.

\section{REDSHIFTS}

\subsection{Redshift estimation}

The main parameter to be determined from the spectra was the redshift of each object. A sophisticated and highly tuned redshift code was developed in order to achieve a high level of precision, reliability and automation in the measurement of redshifts from the 2dFGRS spectral data.

The redshift code (version 990505) uses two quasi-independent automated methods: 'absorption' redshifts are obtained by crosscorrelation with template spectra (after clipping emission lines), and 'emission' redshifts are obtained by finding and fitting emission lines. These automated redshift estimates are followed by a visual check and (occasionally, where necessary) a 'manual' redshift obtained by fitting identified spectral features.

B efore these redshift estimates are made, however, the spectra are pre-processed in a number of ways. Residual features from the strong atmospheric emission lines at 5577, 5893, 6300 and $7244 \AA$ are masked by interpolating over a small wavelength range (20-30A ) centred on the sky line. The atmospheric absorption bands around $6870 \AA$ ( $B$-band) and $7600 \AA$ ( $A$-band), and the fibre absorption band around $7190 \AA$, are removed by dividing by an approximate band correction. This correction is obtained by fitting a low-order polynomial to the mean of all the spectra in the reduced image, dividing the mean spectrum by this smooth fit, finding the region about each band centre where this ratio is less than unity, and setting the band correction to the value of this ratio in these regions, and to unity elsewhere. The spectra, which are not fluxcalibrated, are finally multiplied by a simple quadratic approximation to the mean flux calibration correction in order to give appropriate weighting across the whole spectral range.

An 'absorption' redshift is obtained by cross-correlating the galaxy spectrum against a suite of eight template spectra, following the general method of Tonry \& Davis (1979). The templates that are used cover a broad range of spectral types, and include five galaxies and three stars. The galaxies (and their morphological types) are: 1. NGC 3379 (E), 2. NGC 4889 (CD), 3. NGC 5248 (Sbc), 4. NGC $2276(\mathrm{Sc})$ and 5. NGC $4485(\mathrm{Sm} / \mathrm{Im})$; the stars (and their spectral types) are: 6. HD 116608 (A 1V), 7. HD 23524 (K OV) and 8. BD $05^{\circ} 1668$ (M5V). The galaxy spectra come from spectrophotometric atlas of Kennicutt (1992); the stellar spectra come from the library of J acoby, Hunter \& Christian (1984). The full set of template spectra are shown in Fig. 6.

The galaxy and template spectra are prepared for crosscorrelation using the following steps: (1) continuum-subtraction using a sixth-order polynomial fit to the continuum; (2) removal of strong emission lines by patching out all points more than 5 times the rms variation above the mean; (3) rebinning to a logarithmic wavelength scale with 2048 pixels; (4) apodizing (tapering the ends of the spectrum to zero) by multiplying the first and last 5 per cent of the spectrum with a cosine bell; (5) Fourier transformation and, finally, (6) multiplication by an exponential filter of the form $e^{-k / k_{h}}-e^{-k / k_{1}}$, where $k$ is wavenumber in inverse pixels, $k_{h}=$ 300 pixel $^{-1}$ and $k_{l}=15$ pixel $^{-1}$, in order to reduce the effects of both the residual continuum at low wavenumber and the noise at high wavenumber.

The cross-correlation function is then computed as the inverse Fourier transform of the complex product of the filtered Fourier

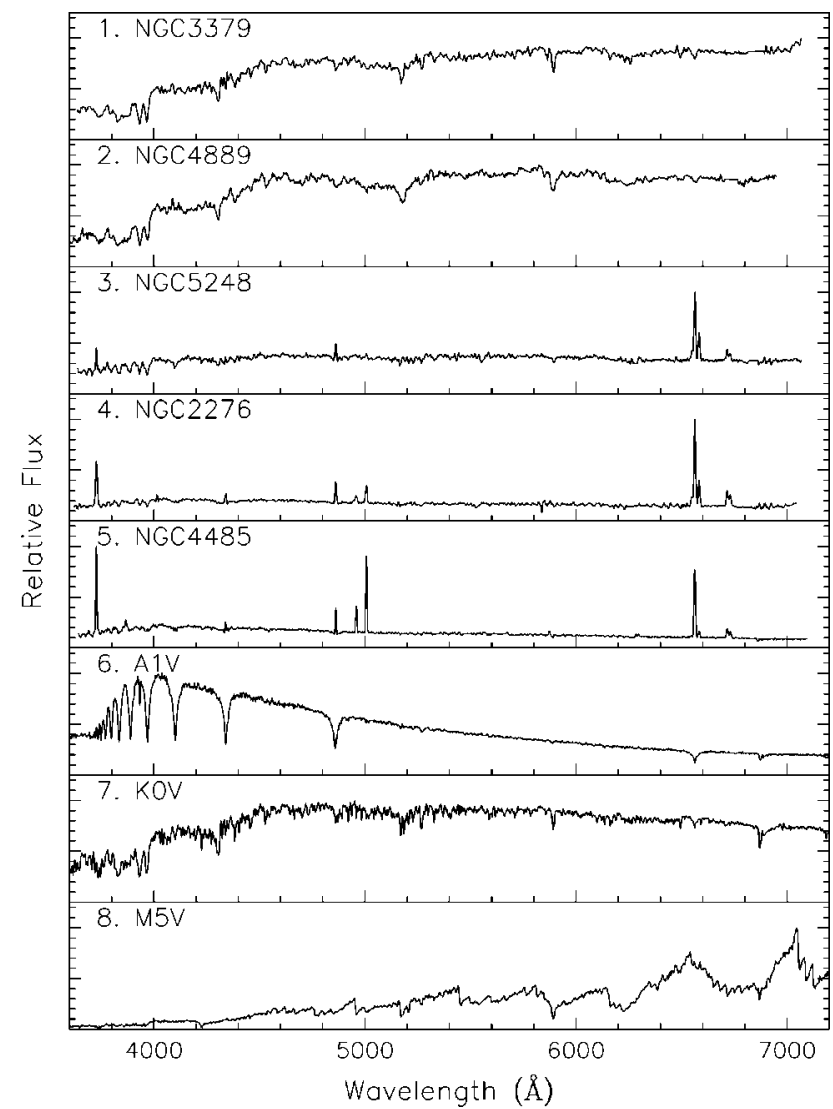

Figure 6 . The eight template spectra against which the $2 \mathrm{dFGRS}$ galaxy spectra were cross-correlated.

transforms of the processed galaxy and template spectra. The highest peak in the cross-correlation function is fitted with a quadratic in order to obtain the position and height of the peak. The significance of the peak is measured in terms of the ratio, $R$, of the height of the peak to the noise in the cross-correlation function, which is estimated from its antisymmetric part. The absorption redshift is taken to be the cross-correlation redshift obtained with the template giving the highest value of $R$. An estimate of the quality of the absorption redshift, $Q_{a}$, is based on the value of $R$, with $Q_{a}=1,2,3,4$ for $R>3.5,4.0,4.5,5.0$ and $Q_{a}=0$ otherwise (with the additional requirement that $Q_{a}=3$ and $Q_{a}=4$ also require, respectively, at least four and six of the eight templates give the same redshift to within $600 \mathrm{~km} \mathrm{~s}^{-1}$ ).

An 'emission' redshift is obtained by fitting Gaussians to significant spectral features and searching for a multiline match. The galaxy spectrum is first continuum-subtracted using a sixthorder polynomial fit to the continuum and lightly smoothed with a Gaussian kernel having a dispersion of 0.8 pixels. Peaks in the smoothed spectrum above a threshold (set at 3.3 times the robustly estimated rms variation) are flagged as candidate emission lines. For each of these, in descending order of strength, a Gaussian is then fitted to the unsmoothed data, using the peak in the smoothed spectrum as the initial guess for the line centre. The Gaussian fits to the stronger lines are subtracted in turn before the weaker lines are fitted (to minimize blending effects in the $\mathrm{H} \alpha / \mathrm{N}$ II pair). Then lines with fitted FWHM between 0.7 and 7 pixels, and total 'significance' of the whole line above $3.5 \sigma$ are marked as 'good'; this rejects unclipped cosmic rays and low-significance features. 
The 'good' lines are then sorted by strength, and the three strongest tested to see if at least two can be identified as common emission lines ([0 ॥] $3727 \AA, H \beta 4861 \AA$, [0 III] $5007 \AA$, $\mathrm{H} \alpha 6563 \AA$ or [ $\mathrm{N} \mathrm{II]} 6584 \AA$ ) at a single redshift (the two-out-ofthree criterion is used to allow for one spurious feature, such as a residual sky line or cosmic ray). If so, then all the other lines in this set (plus [0 III] 4959 A) that match this redshift to better than $600 \mathrm{~km} \mathrm{~s}^{-1}$ are found, and the mean redshift from these matching lines is adopted as the emission redshift. If not, then up to two possible single-line redshifts are kept for comparison with the absorption redshift, assuming that the single line is either [0 $\mathrm{II}$ ] or $\mathrm{H} \alpha$ in the range $0<\mathrm{z}<0.4$, as appropriate. An estimate of the quality of the emission redshift, $Q_{e}$, is based on the number and strength of the lines from which it is determined, with $Q_{e}=0$ if there are no lines, $Q_{e}=1$ for one weak line, $Q_{e}=2$ for any two lines or one strong line, and $Q_{e}=4$ for three or more lines.

The penultimate step is the choice of the best automated redshift estimate, given the absorption and emission redshifts and their quality parameters. The best redshift is taken to be whichever of the absorption and emission redshifts has the higher quality parameter (with the absorption redshift preferred if $Q_{a}=Q_{e}$ ). The best estimate of the redshift quality is $Q_{b}=\max \left(Q_{a}, Q_{e}\right)$, with two exceptions: (i) if the difference between the absorption and emission redshifts is less than $600 \mathrm{~km} \mathrm{~s}^{-1}$, then $Q_{b}=$ $\max \left(Q_{a}, Q_{e}, 3\right)$, and (ii) if $Q_{a} \geq 2$ and $Q_{e} \geq 2$ and the difference between the absorption and emission redshifts is greater than $600 \mathrm{~km} \mathrm{~s}^{-1}$, then this discrepancy is flagged and we set $Q_{b}=1$.

The final step in measuring the redshift is a visual check of the best automatic redshift. The redshift code displays for the user both the cross-correlation function and the galaxy spectrum with all the common spectral features superposed at the best automatic redshift. The spectrum displayed is the version actually used for the redshift estimate, with bad pixels and the strongest night sky lines patched out, and with an approximate correction applied for the instrumental response. To assist in identifying spurious features, the code also displays the mean sky spectrum (to show the positions of the sky features), the atmospheric absorption bands and the variance array associated with the spectrum. The user is given the emission- and/or absorption-line redshifts, the bestestimate redshift, whether this is an emission or absorption redshift (or both), and whether (if both are obtained) the emission and absorption redshifts agree or not. If neither of the automated estimates are correct, the user can manually estimate a redshift by identifying a particular spectral feature and fitting Gaussians at the positions of all the common features. These fits can be to emission or absorption features, and are rejected if there is no detectable feature near the nominal position. The manual redshift is taken to be the mean of the redshifts estimated from each of the well-fitted features. Given all this information, the observer can visually inspect the spectrum, try al ternative redshifts (absorption, emission and manual) and determine the best redshift estimate.

Once the final redshift estimate has been decided, the user assigns a quality, $Q$, to this redshift on a five-point scale, with the nominal interpretation (see below for further discussion): $Q=1$ means no redshift could be estimated; $Q=2$ means a possible, but doubtful, redshift estimate; $Q=3$ means a 'probable' redshift (notionally 90 per cent confidence); $Q=4$ means a reliable redshift (notionally 99 per cent confidence); $Q=5$ means a reliable redshift and a high-quality spectrum. Note that this quality parameter is determined entirely by the subjective judgement of the user, and is independent of the automatic quality parameter $Q_{b}$. Quality classes 1 and 2 are considered failures, so the redshift completeness is the number of $Q=3,4,5$ redshifts divided by the total number of galaxies in the field. The standard redshift sample comprises objects with $Q \geq 3$, but (for applications where redshift reliability is more important than completeness) one may prefer to use the set of objects with $Q \geq 4$.

For the objects ending up with 'acceptable' $(Q=3,4,5)$ redshifts, the best automated redshift is the same as the best visual redshift about 93 per cent of the time; about 5 per cent of the time either the absorption or the emission redshift agrees with the best visual redshift, but the code has preferred the other one; about 2 per cent of the time neither of the estimates matches the best visual redshift, and a manual redshift estimate is required.

While there is some element of subjectivity in deciding whether a redshift estimate is 'acceptable' or not, this visual assessment is found to improve the reliability of the final sample, since the observer can assess a number of factors that are hard to fully automate, such as the overall shape of the spectrum, line ratios, the general quality of sky subtraction, and the proximity of features to sky lines or absorption bands. For 15000 spectra, two or more users have measured redshifts independently using the same redshift code on the same spectra; the pair-wise 'blunder' rate (see below) is 0.4 per cent, which is several times smaller than that for different observations of the same object. The rms difference in redshift completeness (fraction of objects per field with $Q \geq 3$ ) betw een two users for the same data is less than half of that between the same observer measuring redshifts for many different fields. The human variation is therefore substantially smaller than the unavoidable variation between fields due to weather and other effects, and will to first order be corrected by the completeness factors.

\subsection{Quality of spectra}

A quantitative overall measure of spectral quality is the median signal-to-noise ratio $(\mathrm{S} / \mathrm{N})$ per pixel computed over all pixels in the wavelength range $4000-7500 \AA$ with fluxes greater than zero. This is computed directly from the spectrum and it associated variance array, and can be compared to the quality class $Q$ assigned to the redshift identification. The distribution of $\mathrm{S} / \mathrm{N}$ over the five quality classes for the best spectrum of each object (i.e., the spectrum on which the adopted redshift is based) is shown in Fig. 7. The fraction

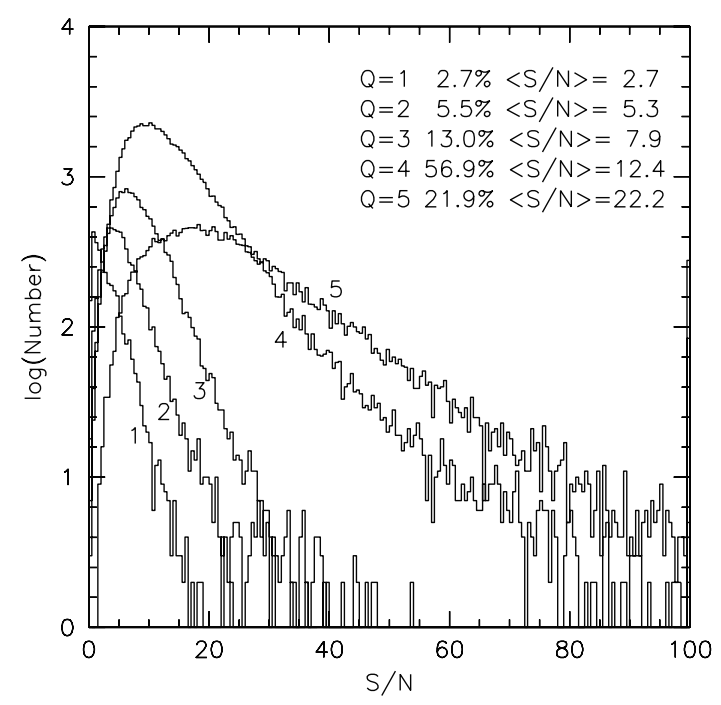

Figure 7. The distribution of the median $\mathrm{S} / \mathrm{N}$ per pixel for objects in each of the five quality classes. 


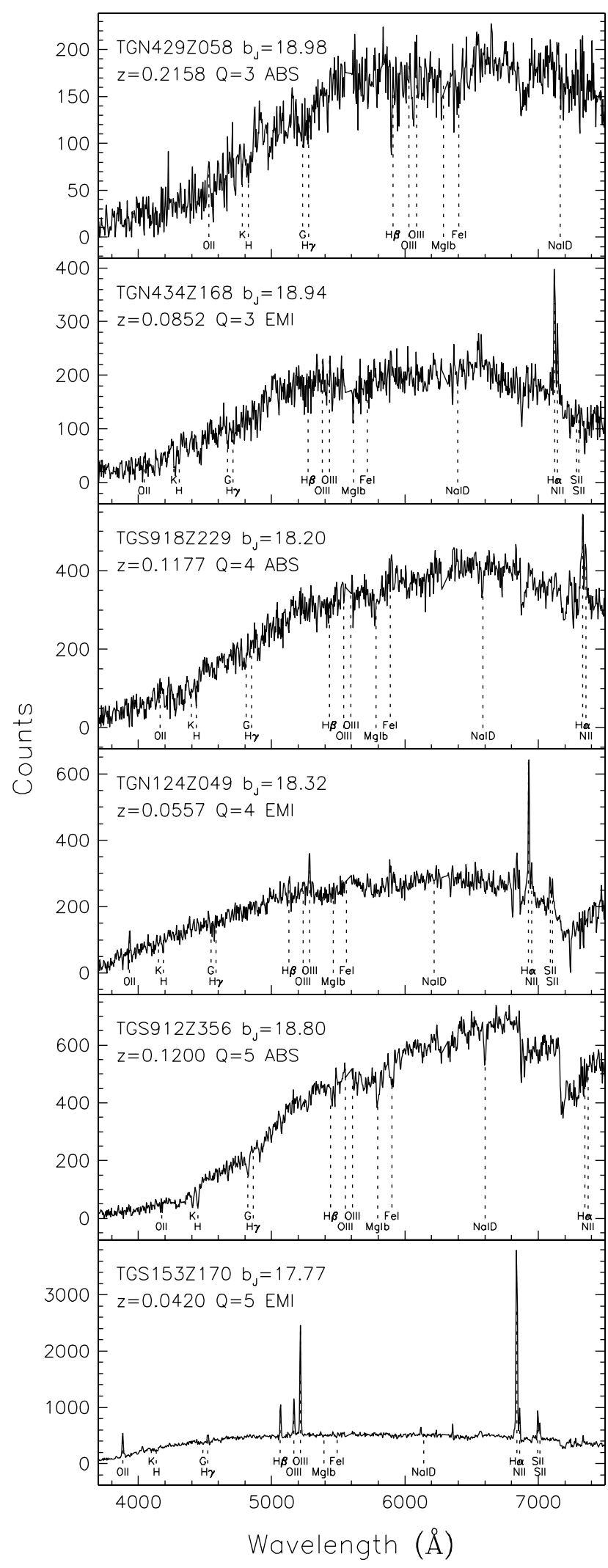

Figure 8. Example 2dFGRS spectra, showing objects with quality classes $Q=3, Q=4$ and $Q=5$, and both absorption (ABS) and emission (EMI) redshifts. The most common spectral features are marked at the wavelengths corresponding to the measured redshift of each object. of objects in each quality class and the median $\mathrm{S} / \mathrm{N}$ for each quality class are given in the figure legend. Objects with reliable redshifts $(\mathrm{Q}=3,4,5)$ make up 91.8 per cent of the sample and have a median S/N of 13.1 pixel $^{-1}\left(6.3 \AA^{-1}\right)$.

Example $2 \mathrm{dFGRS}$ spectra for quality classes $\mathrm{Q}=3, \mathrm{Q}=4$ and $Q=5$ are shown in Fig. 8; for each quality class, one object with an absorption redshift and one object with an emission redshift are shown. In order to be representative, the spectra are chosen to have a $\mathrm{S} / \mathrm{N}$ per pixel close to the median of their quality class. R esiduals from the strong night sky lines at 5577 and $6300 \AA$ have been interpolated over.

\subsection{Repeat observations}

A mongst the first 150000 galaxies in the survey with redshifts, there are 8480 repeat redshift measurements, i.e., a second, or even a third, reliable $(Q=3,4,5)$ redshift measurement for an object. These repeat measurements are either objects that lie in the overlaps between fields (7444 cases) or are in fields which were observed more than once, usually because the first observation was of poor quality and low completeness (1036 cases). For these objects we can compare their best redshift measurement with the repeat measurements to estimate both the rate of incorrect redshift identifications in the survey and the uncertainties on the correct redshift identifications.

The top panel of Fig. 9 shows the distribution of the redshift differences (with respect to the best redshift measurement for the object) for all the repeat observations. The rms redshift difference (robustly estimated here and throughout as the half-width of the central 68 per cent of the distribution) is $120 \mathrm{~km} \mathrm{~s}^{-1}$, implying an overall single-measurement rms uncertainty of $85 \mathrm{~km} \mathrm{~s}^{-1}$.

The lower panels of Fig. 9 show the trends in the redshift uncertainty as a function of redshift, magnitude, $\mathrm{S} / \mathrm{N}$ and quality class $Q$. A s expected, the uncertainties increase at higher redshifts (from $95 \mathrm{~km} \mathrm{~s}^{-1}$ for $z<0.05$ to $150 \mathrm{~km} \mathrm{~s}^{-1}$ at $\mathrm{z}>0.15$ ), fainter magnitudes (from $100 \mathrm{~km} \mathrm{~s}^{-1}$ for $b_{\jmath}<18.5$ to $140 \mathrm{~km} \mathrm{~s}^{-1}$ for $b_{\jmath}>$ 19.3) and lower S/N (from $95 \mathrm{~km} \mathrm{~s}^{-1}$ for $\mathrm{S} / \mathrm{N}>25$ to $145 \mathrm{~km} \mathrm{~s}^{-1}$ for $S / N<5$ ). The strongest trends are with redshift quality class and the method of estimating the redshift. Table 1 gives the rms precision of single measurements as a function of redshift quality class or measurement method $(A B S=$ cross-correlation of absorption features; $E M I=$ automatic fit to emission lines; MAN = manual fit to features). The fact that manual redshift estimation has the largest uncertainties mainly reflects the fact that it is only employed when the automatic methods have failed, usually in cases of low $\mathrm{S} / \mathrm{N}$.

We divide the repeat measurements into two categories: correct identifications, where the redshift difference is less than $600 \mathrm{~km} \mathrm{~s}^{-1}$, and 'blunders', where it is greater than $600 \mathrm{~km} \mathrm{~s}^{-1}$. The division at $600 \mathrm{~km} \mathrm{~s}^{-1}$ corresponds to 5 times the rms error in the redshift differences. It should be noted that these blunders are not necessarily due to incorrect redshift measurements for one (or both) of the observed spectra. They may also be due to two observations of a close pair of objects with slight offsets in the fibre position, resulting in a different member of the pair dominating the spectral flux in each observation.

There are 263 blunders, so that the pair-wise blunder rate amongst the $Q=3,4,5$ repeat measurements is 3.1 per cent. This implies a single-measurement blunder rate of just 1.6 per cent. Fig. 10 shows plots of the redshift differences for the blunders with respect to redshift, magnitude, $\mathrm{S} / \mathrm{N}$ and quality class; it also shows 

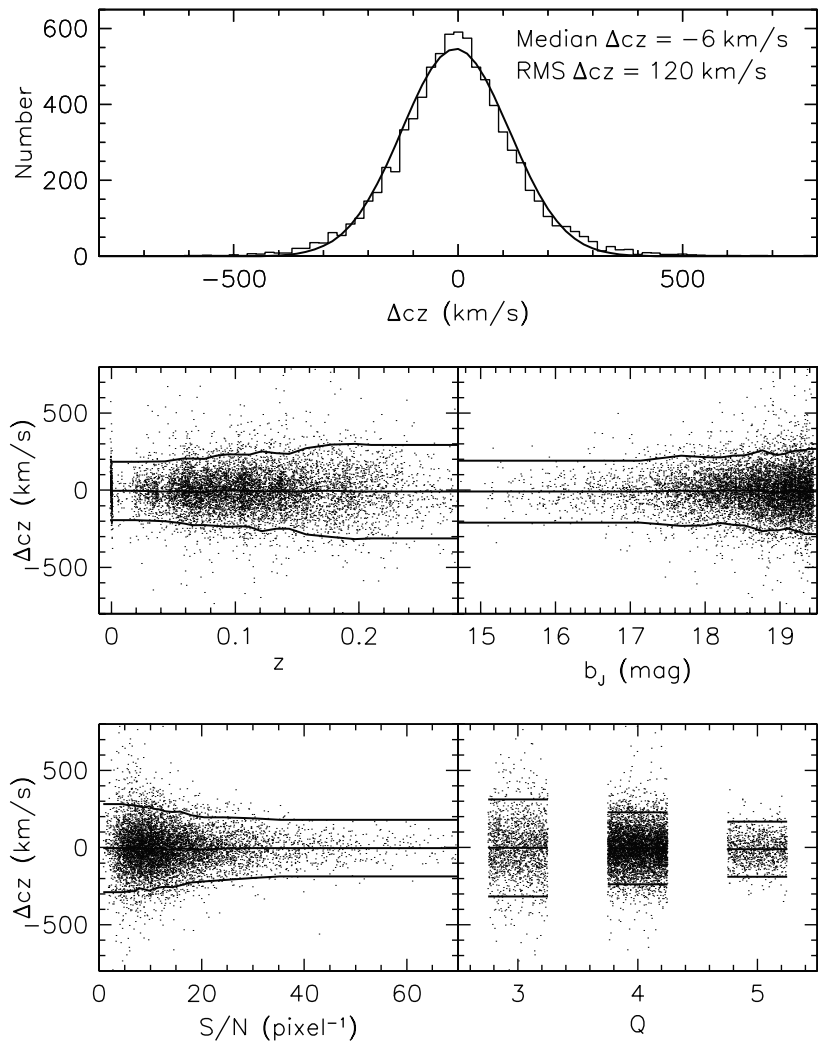

Figure 9. The distribution of redshift differences between repeat measurements with $Q \geq 3$ is shown in the top panel, along with the Gaussian having the same median and $\mathrm{rms}$. The dependence of the redshift differences on redshift, apparent magnitude, $\mathrm{S} / \mathrm{N}$ ratio and quality class for the best observation, is shown in the lower panels (note that the quality classes have small random offsets added to make the distribution of redshift differences clear). The lines in the lower panels are the running median and the $\pm 2 \sigma$ ranges.

Table 1. Summary statistics by quality class and method.

\begin{tabular}{lccc}
\hline $\begin{array}{l}\text { Quality } \\
\text { class }\end{array}$ & $\begin{array}{c}\text { Fraction } \\
\text { of sample }\end{array}$ & $\begin{array}{c}\text { rms error } \\
\left(\mathrm{km} \mathrm{s}^{-1}\right)\end{array}$ & $\begin{array}{c}\text { Blunder } \\
\text { rate }\end{array}$ \\
\hline & & & \\
$Q=1$ & $2.5 \%$ & - & - \\
$Q=2$ & $4.8 \%$ & 143 & $69.5 \%$ \\
$Q=3$ & $11.9 \%$ & 123 & $10.2 \%$ \\
$Q=4$ & $57.3 \%$ & 89 & $0.9 \%$ \\
$Q=5$ & $23.5 \%$ & 64 & $0.2 \%$ \\
Redshift & Fraction & rms error & Blunder \\
method & of sample & $\left(\mathrm{km} \mathrm{s}^{-1}\right)$ & rate \\
& & & - \\
$Q=1,2$ & $7.3 \%$ & - & - \\
ABS & $72.0 \%$ & 87 & $0.7 \%$ \\
EM I & $18.3 \%$ & 61 & $0.9 \%$ \\
MAN & $2.4 \%$ & 159 & $9.3 \%$ \\
\hline
\end{tabular}

the pair-wise fraction of blunders as a function of each of these quantities.

There is no increase in the blunder rate with redshift, but a significant increase at fainter magnitudes, lower $\mathrm{S} / \mathrm{N}$ and lower quality class. The single-measurement blunder rates as functions of quality class and measurement method are given in Table 1 . We
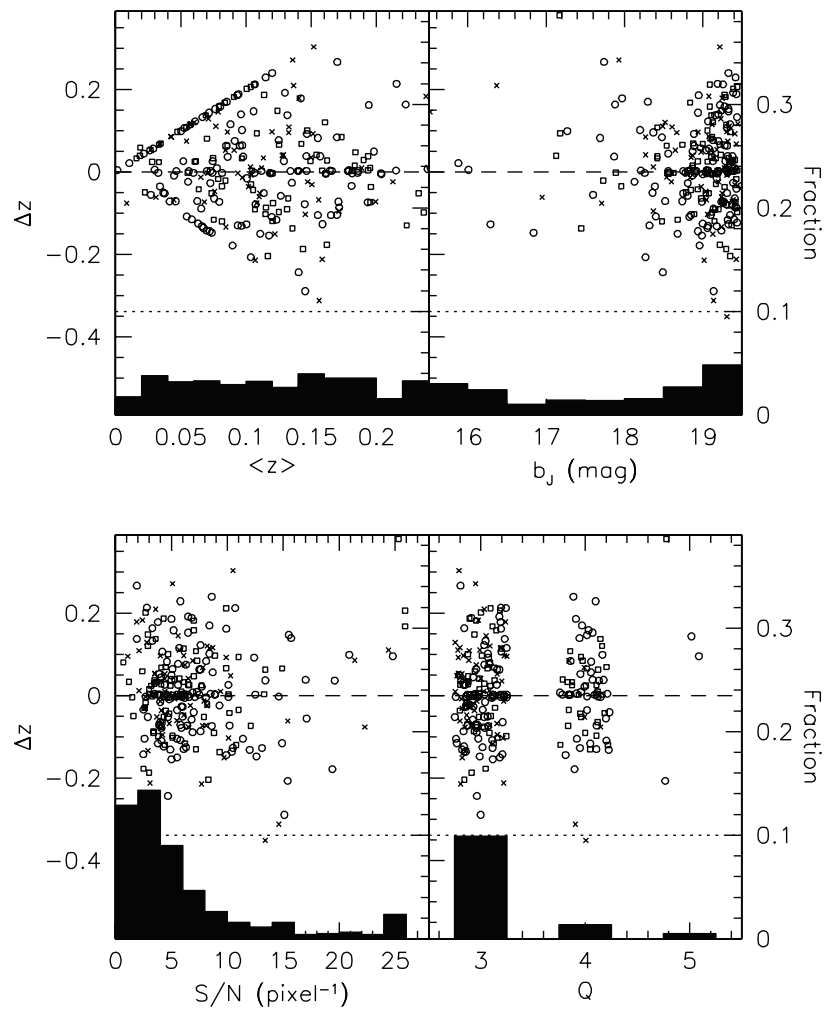

Figure 10. The distribution of the redshift differences for the blunders (repeat observations with $|\Delta \mathrm{cz}|>600 \mathrm{~km} \mathrm{~s}^{-1}$ ) against redshift, magnitude, $\mathrm{S} / \mathrm{N}$ and quality class. The circles are absorption redshifts, the squares are emission redshifts and the crosses are manual redshifts. The histograms at the foot of each panel show the fraction of blunders as a function of redshift, magnitude, $\mathrm{S} / \mathrm{N}$ and quality class.

find that $Q=3$ corresponds to individual redshifts having 90 per cent reliability, and that $Q=4$ and 5 correspond to better than 99 per cent reliability, as intended. The blunder rate for $Q=2$ redshifts is 70 per cent, consistent with the definition of $Q=2$ as 'possible but doubtful'. The blunder rates for the absorption and emission redshifts are less than 1 per cent, but the blunder rate for manual redshifts is 9 per cent (due to the fact that manual redshifts are used only in the 2.4 per cent of cases where the automatic methods fail).

The distribution of blunders shows a slight peak around zero redshift difference, suggesting that the tail of the error distribution for correct redshift identifications is more extended than a Gaussian. Some of the blunders may thus be correct identifications with larger than normal errors, implying that we may be slightly over-estimating the blunder rate. However, the most notable feature in the distribution of blunders is the large number of instances (22 per cent of the total) where one or other of the redshifts in the comparison is approximately zero. The redshift difference is defined as (other redshift - best redshift), so the upper envel ope corresponds to objects where the best redshift is zero and the lower envel ope corresponds to the other redshift being zero. In the former case the best redshift indicates that the objects are stars and the other (non-zero) redshift is due to a blunder based on a poor-quality spectrum; in the latter case we have the reverse of this situation. There are 3 times as many objects in the upper envelope as the lower, so stars being mistaken for galaxies is a much more common error than galaxies being mistaken for stars - which is remarkable, given that galaxies are 20 times more common than stars in our sample. 
We have repeated the above analyses after separating the repeat observations into those cases where the repeat is due to re-observing a field (12 per cent) and those where it is due to observing an object in overlapping fields ( 88 per cent). We find no significant difference in either the single-measurement rms precision or the blunder rate for these two subsets of repeat measurements.

Examination of the individual object spectra in the cases where there is a blunder shows that in general the best redshift appears to be quite reliable, and that the main reason for the discrepancy is that the other redshift measurement has been obtained from a poorquality (low-Q) spectrum.

\subsection{External comparisons}

In order to compare our redshift measurements with those from other sources, we have matched our catalogue to the redshift catalogues from the second Center for A strophysics redshift survey (CfA 2) (Huchra, Vogeley \& Geller 1999), the Stromlo-APM redshift survey (SAPM) (Loveday et al. 1996), the PSCz survey (PSCz) (Saunders et al. 2000), the Las Campanas redshift survey (LCRS) (Shectman et al. 1996), and also to the heterogeneous redshift compilation of the ZCAT catalogue (version 2000 November 13; J. P. Huchra, private communication). Objects in these catalogues were matched to objects in the $2 \mathrm{dFGRS}$ by position; positions separated by 4 arcsec or less were assumed to belong to the same object. This criterion was chosen to include most genuine matches and exclude most false matches between close but unrelated objects.

Table 2 and Fig. 11 summarize the results of these comparisons. The table lists, for each external source catalogue, the number of matched objects in the comparison, $\mathrm{N}_{\text {comp; }}$ the number of cases, $\mathrm{N}_{600}$, where the redshift difference between the $2 \mathrm{dFGRS}$ and the other source indicates a blunder $\left(|\Delta \mathrm{cz}|>600 \mathrm{~km} \mathrm{~s}^{-1}\right)$, the median redshift difference, $\langle\Delta c z\rangle$, and the rms scatter of $\Delta c z$ about this median, $\sigma(\Delta c z)$. The median and rms scatter are estimated excluding the blunders.

In interpreting these comparisons we need to keep in mind the magnitude range of the objects that are in common. Fig. 12 shows the magnitude distributions of the comparison samples, all of which are significantly brighter than the $2 \mathrm{dFGRS}$ itself. The CfA 2, SA PM and PSC z catal ogues only overlap with the bright tail, while the median magnitude of the LCRS is a magnitude brighter than that of the $2 \mathrm{dFGRS}$. ZCAT has a broader magnitude range, but is also heavily weighted towards brighter objects.

The best comparison is with the LCRS: the comparison sample is the largest, the magnitude range most similar, and the catalogue is homogeneous (unlike ZCAT). The rms redshift difference of $109 \mathrm{~km} \mathrm{~s}^{-1}$ is consistent with the single-measurement uncertainty of $76 \mathrm{~km} \mathrm{~s}^{-1}$ for objects in the $2 \mathrm{dFGRS}$ brighter than $\mathrm{b}_{\mathrm{j}}=18.5$ and the typical uncertainty of $67 \mathrm{kms}^{-1}$ on the LCRS redshifts (Shectman et al. 1996). The pair-wise blunder rate in the comparison sample is $11 / 3135=0.4$ per cent, which in fact is rather low er than might be expected from the pair-wise blunder rate of $46 / 2920=1.6$ per cent for repeat measurements of objects in the $2 \mathrm{dFGRS}$ brighter than $b_{j}=18.5$; this may be due to the high surface brightness selection criterion of the LCRS.

The worst comparison is with ZCAT: the rms redshift difference is $127 \mathrm{~km} \mathrm{~s}^{-1}$, and the pair-wise blunder rate is $103 / 1593=6.5$ per cent, which is more than twice the pair-wise blunder rate for the $2 \mathrm{dFGRS}$ repeats. Given that the singlemeasurement blunder rate for the $2 \mathrm{dFGRS}$ is 1.6 per cent, the
Table 2. Comparisons of redshifts with external sources.

\begin{tabular}{lrrrr}
\hline Source & $\mathrm{N}_{\text {comp }}$ & $\mathrm{N}_{600}$ & $\begin{array}{c}\langle\Delta \mathrm{CCZ}\rangle \\
\mathrm{km} \mathrm{s}^{-1}\end{array}$ & $\begin{array}{r}\sigma(\Delta \mathrm{CZ}) \\
\mathrm{km} \mathrm{s}^{-1}\end{array}$ \\
\hline CfA2 & 87 & 1 & -22 & 93 \\
SAPM & 228 & 10 & +11 & 123 \\
PSCZ & 172 & 7 & -11 & 120 \\
LCRS & 3135 & 11 & -1 & 109 \\
ZCAT & 1593 & 103 & -33 & 127 \\
\hline
\end{tabular}

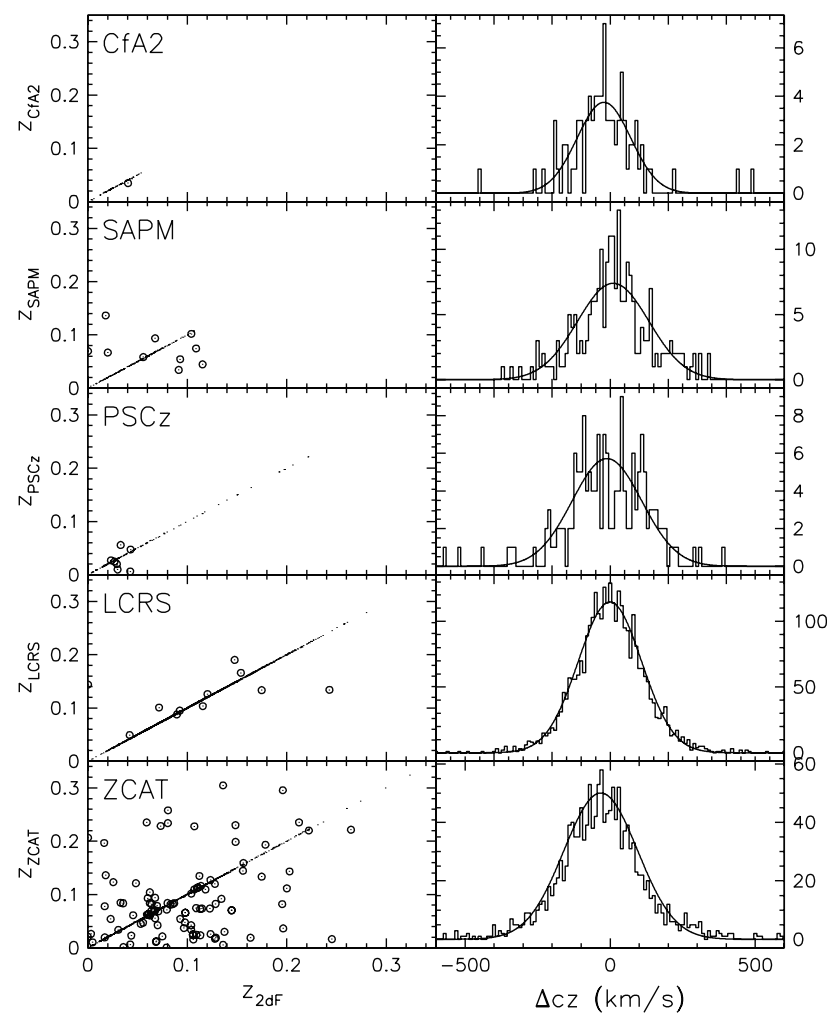

Figure 11. Comparisons of the $2 \mathrm{dFGRS}$ redshifts with those from the CFA 2, SA PM , LCRS and ZCAT catal ogues. The left-hand panels shown the correlations between the redshift measurements, with each comparison shown as a dot. Most dots lie along the 1-to-1 line; the relatively few blunders $\left(|\Delta \mathrm{cz}|>600 \mathrm{~km} \mathrm{~s}^{-1}\right)$ are circled. The right-hand panels show the histogram of redshift differences overlaid with a Gaussian having the same median and rms.

implied single-measurement blunder rate for ZCAT is 5.0 per cent, which is presumably due to the heterogeneous nature of its source catalogues.

A lthough the samples in common with CfA 2, SAPM and PSCz are relatively small, they give results that are consistent with the rms redshift errors and pair-wise blunder rate obtained from the $2 \mathrm{dFGRS}$ repeat observations.

Checking the $2 \mathrm{dFGRS}$ spectra of the objects that have redshift blunders, we find that 0/1 cases in the comparison with CfA 2, 1/10 for SAPM , 1/7 for PSCz and 4/11 for LCRS might possibly be attributed to errors in the $2 \mathrm{dFGRS}$ redshift estimates. Examining the DSS images of these objects, we find that two of the discrepancies with SAPM, one of those with PSC $z$, and six of those with LCRS, occur in cases where there is a close companion to the target object and hence some potential for confusion. 


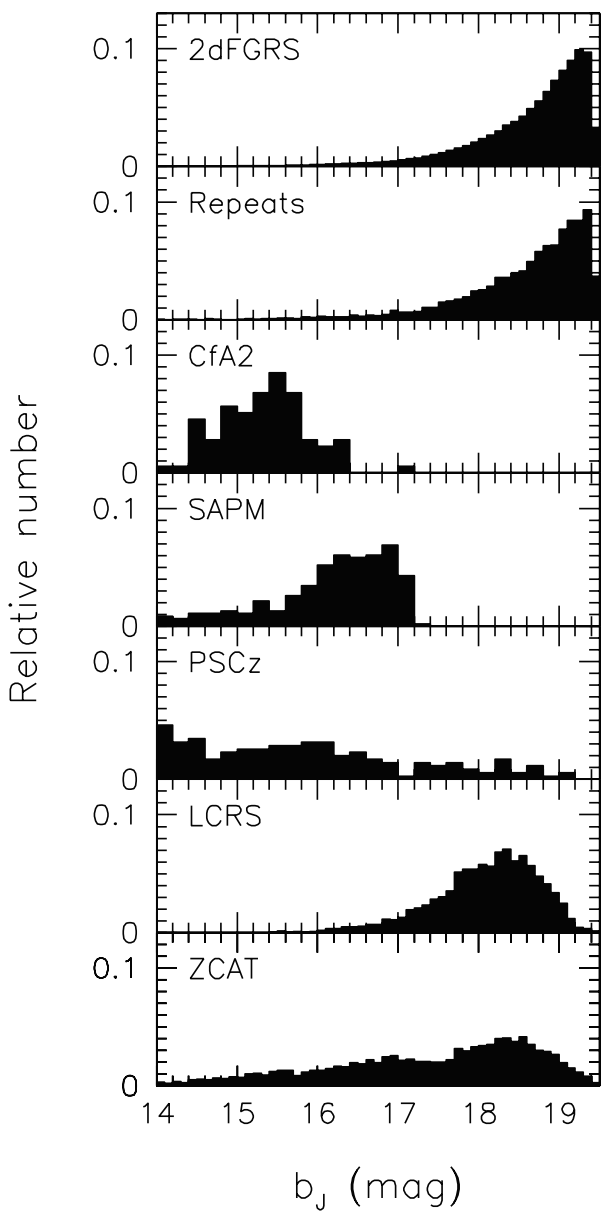

Figure 12. The magnitude distributions of the $2 \mathrm{dFGRS}$ sample, the repeat observations in the 2dFGRS sample, and the five samples of objects in common with other redshift catalogues: CfA2, SAPM, PSC $z, L C R S$ and ZCAT.

\section{SURVEY MASK S}

For accurate statistical analysis of the $2 \mathrm{dFGRS}$ it is essential to fully understand the criteria that define its parent photometric catal ogue and also the spatial and magnitude-dependent completeness of the redshift catalogue. For this purpose we have defined three maps or masks characterizing this information as a function of position on the sky.

(1) The magnitude limit mask gives the extinction-corrected magnitude limit of the survey at each position.

(2) The redshift completeness mask gives the fraction of measured redshifts at each position.

(3) The magnitude completeness mask gives a parameter defining how the redshift success rate depends on apparent magnitude.

Each mask has its own use, but for some analyses it is necessary to make use of two or even all three masks. We now describe in more detail how each one of these masks is defined, and briefly outline some of their uses.

\subsection{Magnitude limit mask}

Although the $2 \mathrm{dFGRS}$ sample was originally selected to have a uniform extinction-corrected magnitude limit of $b_{j}=19.45$, in fact the survey magnitude limit varies slightly with position on the sky. There are two reasons for this. First, the photometric calibrations now available are much more extensive than when the parent $2 \mathrm{dFGRS}$ catalogue was originally defined. This has enabled us to recalibrate the whole $2 \mathrm{dFGRS}$ parent catalogue ( $M$ addox et al., in preparation), and results in new zero-point offsets and linearity corrections for each of the UK ST photographic plates. Second, the extinction corrections have been changed to use the final published version of the Schlegel et al. (1998) extinction maps; the original extinction corrections came from a preliminary version of those maps.

The magnitude limit mask is therefore defined by the change in the photometric calibration of each UK ST photographic plate and the change in the dust extinction correction at each position on the sky. The magnitude limit masks for the NGP and SGP strips are shown in Fig. 13; note that the mask also accounts for the holes in the source catalogue around bright stars and plate flaws.

In the SGP, which is a subset of the APM galaxy survey ( $M$ addox et al. 1990a,b,c), the rms change in plate zero-point is only 0.03 mag. How ever, in the NGP region the original calibration was less accurate, and the change in zero-points have an rms of $0.08 \mathrm{mag}$. The change in the dust corrections are also less in the $S G P$, as the extinction is generally lower in this region. In the SGP the rms magnitude change due to improved dust corrections is 0.01 mag, while in the NGP it is 0.02 mag.

The magnitude limit distribution over the NGP and SGP strips is shown in Fig. 14. In the SGP the median limiting magnitude is $b_{j}=19.40$, with an rms about this value of 0.05 mag; in the NGP the median limiting magnitude is $b_{j}=19.35$, with an $r m s$ of 0.11 mag.

For accurate statistical analysis of the $2 \mathrm{dF}$ survey the magnitude limits defined by this mask should be used. It is always possible to analyse the data with a fixed magnitude limit if one is prepared to omit both the areas of the survey that have magnitude limits brighter than the chosen limit and also all the galaxies in the remaining areas with magnitudes fainter than the chosen limit.

\subsection{Simple redshift completeness mask}

The best way to define a redshift completeness mask is to make use of the geometry defined by the complete set of $2^{\circ}$ fields that were used to tile the survey region for spectroscopic observations. Each region of the sky inside the survey boundary is covered by at least one $2^{\circ}$ field, but more often by several overlapping fields. We define a sector as the region delimited by a unique set of overlapping $2^{\circ}$ fields. This is the most natural way of partitioning the sky, as it takes account of the geometry imposed by the pattern of $2^{\circ}$ fields and the way in which the galaxies were targeted for spectroscopic observation. Within each sector, $\theta$, we define the redshift completeness, $R(\theta)$, as the ratio of the number of galaxies for which redshifts have been obtained, $N_{z}(\theta)$, to the total number of objects contained in the parent catalogue, $N_{p}(\theta)$ :

$\mathrm{R}(\theta)=\mathrm{N}_{\mathrm{z}}(\theta) / \mathrm{N}_{\mathrm{p}}(\theta)$.

The redshift completeness of a given sector, $R(\theta)$, should be clearly distinguished from the redshift completeness of a given field, $C_{F}$, since multiple overlapping fields can contribute to a single sector.

This simple redshift completeness mask, shown in Fig. 15, can be used to locate regions in which the redshift completeness is high. It can also be used as a first step either in applying weights to statistically correct for incompleteness, or in constructing a random 

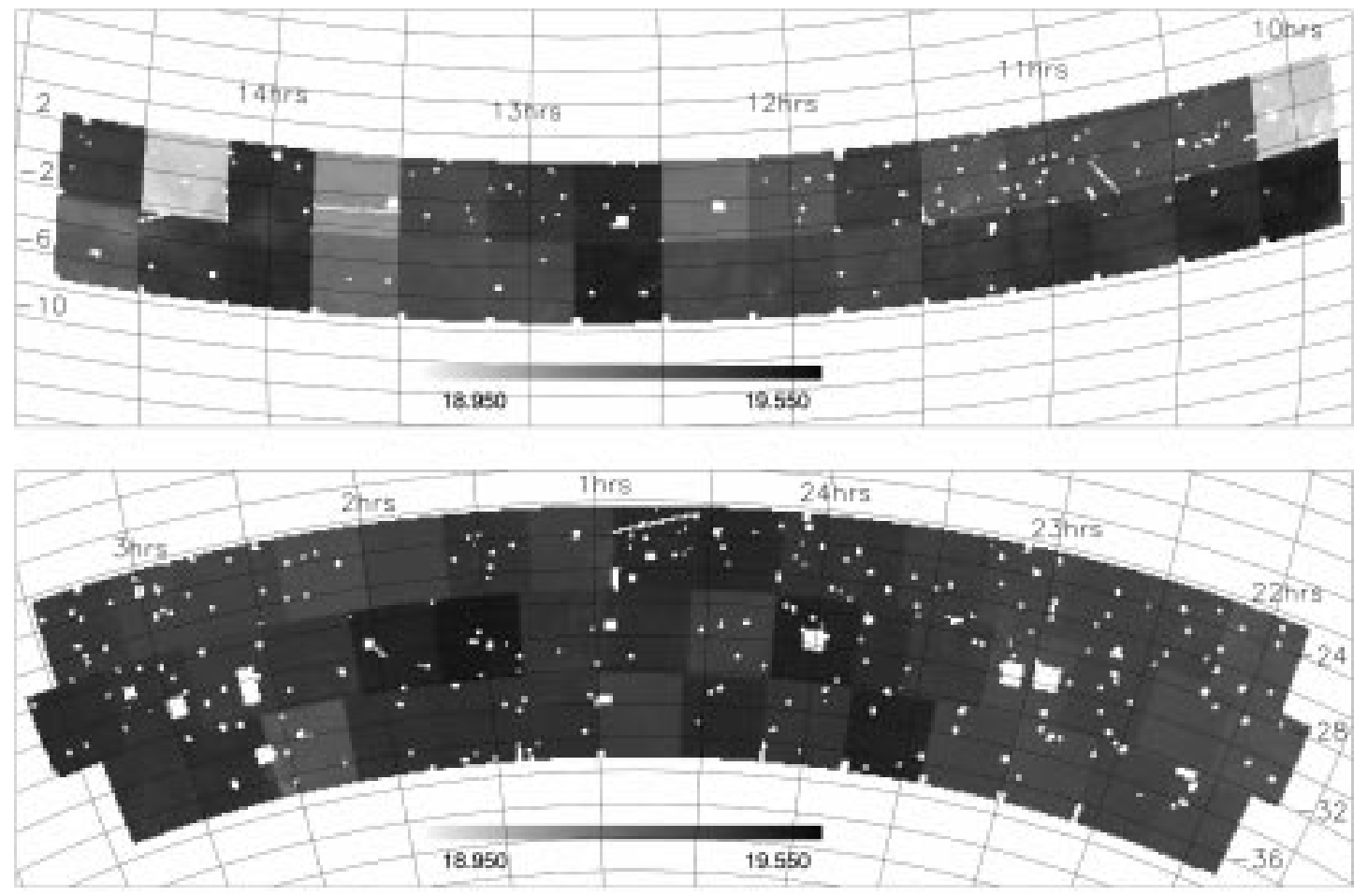

Figure 13. The magnitude limit masks for the NGP strip (top) and the SGP strip (bottom), plotted in a zenithal equal area projection.

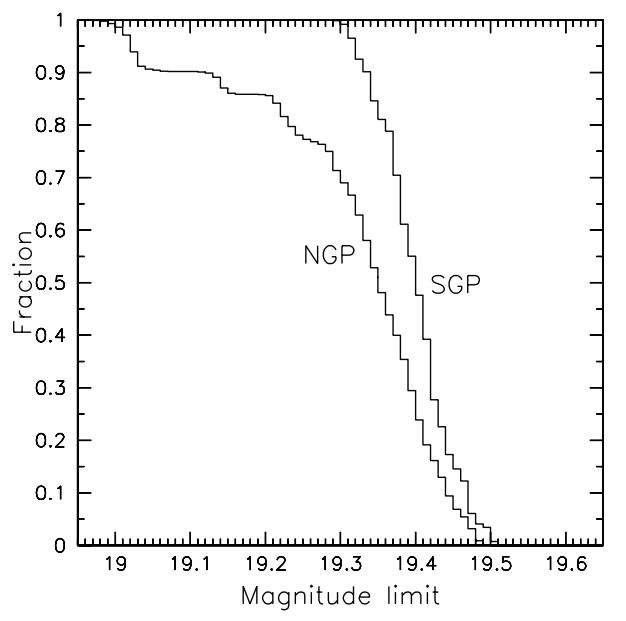

Figure 14. The fraction of the sky in the NGP and SGP survey strips where the survey limit is fainter than a given $b_{j}$ magnitude.

unclustered catalogue (for use in estimating correlation functions) that has the same angular pattern of incompleteness as the redshift sample. For this latter purpose one should also take account of how the redshift completeness depends on position within a sector as a result of constraints on fibre positioning and other considerations. This is best done by using the parent catal ogue to derive weights for each galaxy with a measured redshift (N orberg et al. 2001). A Iso, as discussed in the next section, one should take account of how the redshift completeness depends on apparent magnitude.

\subsection{M agnitude completeness mask}

The success rate of measuring redshifts is generally very high. Fields for which the field completeness, $C_{F}$, is less than 70 per cent are re-observed, while of the remainder just over 76 per cent have a completeness greater than 90 per cent. As one approaches the magnitude limit of the survey, it becomes increasingly difficult to obtain good-quality spectra from which reliable redshifts can be measured. Hence the success of measuring redshifts (the redshift completeness) is a function of apparent magnitude.

In Fig. 16 we show the redshift completeness as a function of apparent magnitude for four different intervals of field completeness, $C_{F}$. We see that in all cases the completeness is a function of apparent magnitude, and that the magnitude at which the completeness begins to drop is brightest in the fields with lowest $C_{F}$, which are generally those taken in marginal observing conditions. Overall, the magnitude-dependent incompleteness is well modelled by a function of the form

$\mathrm{C}_{\mathrm{z}}(\mathrm{m}, \mu)=\gamma[1-\exp (\mathrm{m}-\mu)]$,

with $\gamma=0.99$ and the parameter $\mu$ depending on $C_{F}$. For each field we have chosen to fix $\mu$ by combining equation (5) with a simple power-law model for the galaxy number counts, $N(m) \propto \exp (\alpha m)$, so that $\mathrm{C}_{\mathrm{F}}$ and $\mu$ are related by

$c_{F}(\mu)=\frac{\int_{m_{1}}^{m_{2}} N(m) c_{z}(m, \mu) d m}{\int_{m_{1}}^{m_{2}} N(m) d m}$,

where $m_{1}$ and $m_{2}$ are the bright and faint magnitude limits. This integral can be evaluated to give

$C_{F}(\mu)=\gamma\left[1-g(\alpha) \exp \left(m_{1}-\mu\right)\right]$

where

$g(\alpha)=\frac{\alpha}{\alpha+1} \frac{\exp \left[(\alpha+1)\left(m_{2}-m_{1}\right)\right]-1}{\exp \left[\alpha\left(m_{2}-m_{1}\right)\right]-1}$,

and we take $\alpha=0.5$ as appropriate for the galaxy number counts 

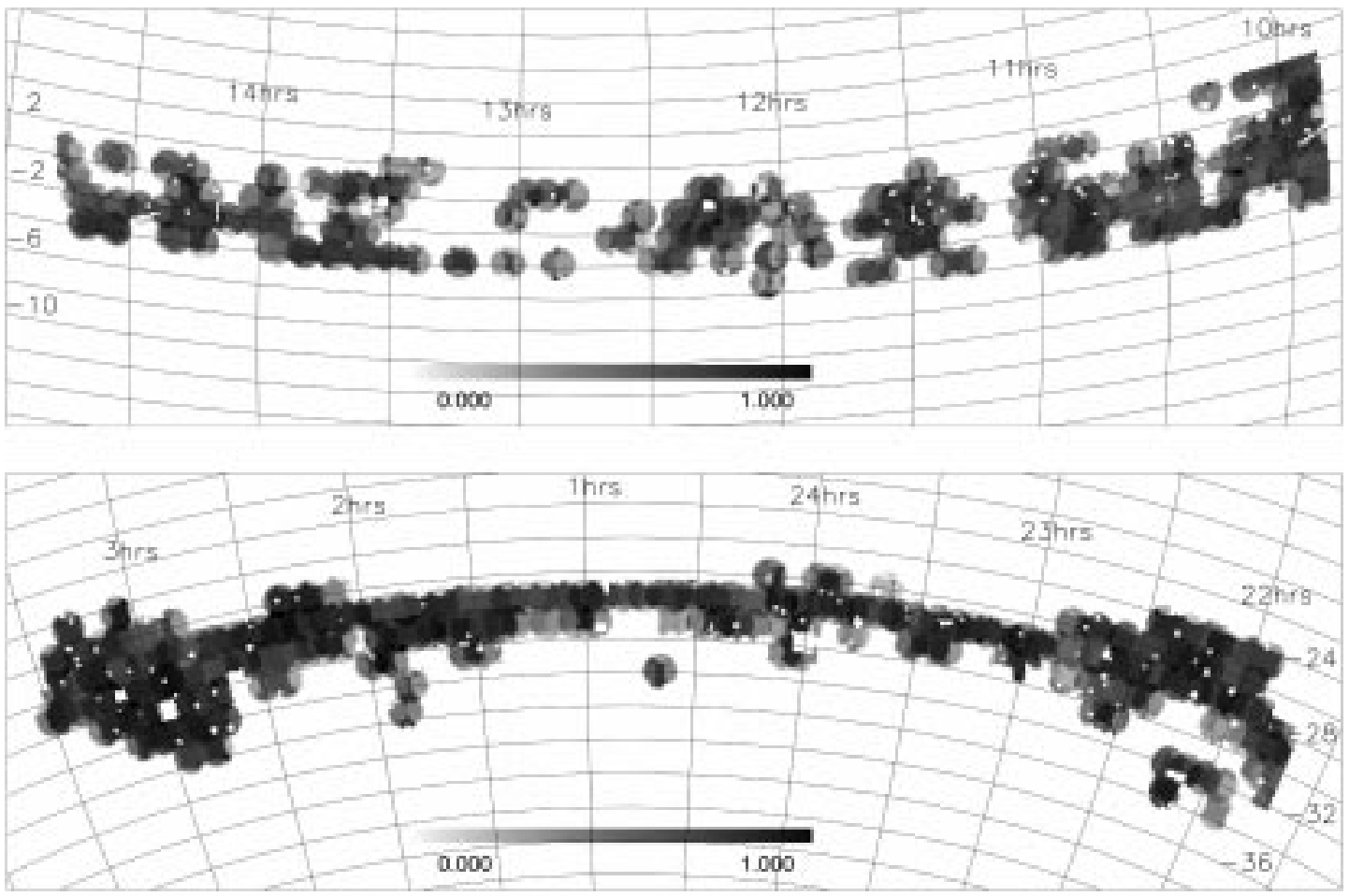

Figure 15. The redshift completeness, $\mathrm{R}(\theta) \equiv \mathrm{N}_{\mathrm{z}}(\theta) / \mathrm{N}_{\mathrm{p}}(\theta)$ as a function of position for the $100 \mathrm{k}$ public release dataset (2dFGRS data from $19970 \mathrm{ctober}$ to 2000 J anuary). The top panel is for the NGP, the bottom panel for the SGP, plotted in a zenithal equal-area projection.
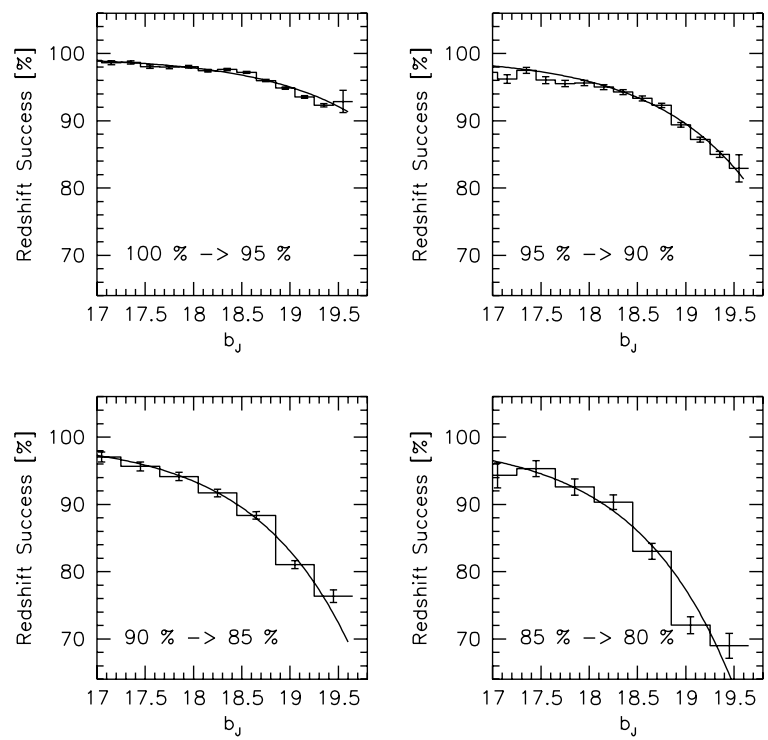

Figure 16. Redshift completeness as a function of apparent magnitude. The different panels are for fields of different overall field completeness: top left, fields with completeness in the range 95 per cent $<c_{F} \leq 100$ per cent; top right, fields with 90 per cent $<c_{F} \leq 95$ per cent; bottom left, fields with 85 per cent $<C_{F} \leq 90$ per cent, and bottom right, fields with 80 per cent $\angle C_{F} \leq 85$ per cent. N ote that over 76 per cent of the observed fields fall in the first two bins, with $c_{F}>90$ per cent. The dashed curves are one-parameter model fits (see text).

around $b_{j}=19$. This equation can be inverted, yielding

$\mu=\ln \left[\frac{g(\alpha) \exp \left(m_{1}\right)}{\left(1-c_{F} / \gamma\right)}\right]$

which enables $\mu$ to be computed for each observed field.
Our goal is to define the value of $\mu$ characterizing the magnitude-dependent completeness for each position in the sky. Since the $2^{\circ}$ fields overlap, it again makes sense to define $\mu(\theta)$ for each sector $\theta$. The value of $\mu(\theta)$ is defined by an appropriately weighted average of the $\mu$ values of the $N_{F}(\theta)$ overlapping observed fields comprising the sector. Specifically, we take

$\mu(\theta)=-\ln \left[\sum_{i=1}^{N_{F}(\theta)} f_{i} \exp \left(-\mu_{i}\right)\right]$,

where $f_{i}$ is the fraction of the observed galaxies in this sector that were targeted in field $i$.

Finally, it is straightforward to combine the magnitude completeness given by $\mu(\theta)$ with the redshift completeness $R(\theta)$ to define an estimate of redshift completeness that depends on both position (sector) and magnitude:

$\mathrm{S}(\theta, \mathrm{m})=\frac{\mathrm{N}_{\mathrm{p}}(\theta)}{\mathrm{N}_{\mathrm{e}}(\theta)} \mathrm{R}(\theta) \mathrm{C}_{\mathrm{z}}[\mathrm{m}, \mu(\theta)]$.

In the first factor, $\mathrm{N}_{\mathrm{p}}(\theta)$ is again the number of parent catalogue galaxies in this sector, while

$\mathrm{N}_{\mathrm{e}}(\theta)=\sum_{\mathrm{i}=1}^{\mathrm{N}_{\mathrm{p}}(\theta)} \mathrm{c}_{\mathrm{z}}\left[\mathrm{m}_{\mathrm{i}}, \mu(\theta)\right]$

is an estimate of the number of galaxies one would expect to have measured redshifts for given the value of $\mu$ that has been assigned to this sector. For ease in calculating equation (11) we tabulate $\mathrm{N}_{\mathrm{p}}(\theta) / \mathrm{N}_{\mathrm{e}}(\theta)$ as well as $\mu(\theta)$ and $\mathrm{R}(\theta)$, using, for convenience, a pixellated mask rather than the exact sectors.

\section{SURVEY DATA BASE}

The 2dFGRS data base consists of three main components: (i) a 
collection of FITS files, one per object in the source catal ogue, that contains every piece of information about each object from the source catalogue, spectroscopic observations and subsequent analysis; (ii) an mSQL data base (Jepson \& Hughes 1998), which contains all of the parameters for each object and allows complex searching and subsetting of the survey objects and the retrieval of selected subsets of spectra and images, and (iii) a W WW interface, which provides a number of different modes for querying the $\mathrm{MSQL}$ data base and a variety of ways for returning the results of such queries. The next three subsections describe these three data base components.

\subsection{Object FITS files}

There are 467214 target objects in the FITS data base, each with its own FITS file. This number is much larger than the number of objects surveyed, since it includes objects down to $b_{j}=19.6$ in the
NGP and SGP strips. Each object in the survey source catalogue has been given a serial number ( $S E Q N U M)$, and the name of the FITS file for that object is this serial number. The serial numbers for objects in the SGP strip are 1- 193550 and 416694-467214, for objects in the NGP strip 193551-332694 and 389714-416693 and for objects in the random fields 332695-389713.

Each FITS file has a primary part which contains all the source catalogue data about the object (as FITS keywords) and a DSS postage stamp image of the object. Spectra are appended as additional FITS extensions. Each spectrum extension contains the spectrum of the object, the variance (error) array for the object spectrum, the spectrum of the mean sky that was subtracted from the object spectrum, and FITS keyword data giving information about the spectroscopic observation and derived parameters such as the redshift and spectral quality. M any targets contain multiple spectrum extensions corresponding to multiple observations.

Table 3 lists all the FITS keywords present in the primary part

Table 3. FITS keywords for the primary image (extension 0).

\begin{tabular}{|c|c|c|}
\hline Keyword & Example & Definition \\
\hline SIMPLE & $\mathrm{T}$ & file does conform to FITS standard \\
\hline BITPIX & 16 & number of bits per data pixel \\
\hline NAXIS & 2 & number of data axes \\
\hline NAXIS1 & 49 & length of data axis 1 \\
\hline NAXIS2 & 49 & length of data axis 2 \\
\hline EXTEND & $\mathrm{T}$ & FITS dataset may contain extensions \\
\hline BSCALE & 1.0000 & $\mathrm{REAL}=(\mathrm{FITS} \times \mathrm{BSCALE})+\mathrm{BZERO}$ \\
\hline BZERO & 0.0000 & zeropoint of conversion to REAL \\
\hline SEQNUM & 100100 & serial number : data base primary key \\
\hline NAME & 'TGS4697164' & 2dFGRS assigned name \\
\hline IMAGE & 'SKYCHART' & existence of postage stamp image \\
\hline RA & 0.7943429758 & RA (B1950) in radians : 323.00 \\
\hline $\mathrm{DEC}$ & -0.5475286941 & DEC (B1950) in radians : -31 2215.9 \\
\hline EQUINOX & 1950.00 & equinox of RA and DEC \\
\hline BJSEL & 18.93 & final bj mag used in the object selection \\
\hline $\mathrm{PROB}$ & 2335.4 & psi star-galaxy classification parameter \\
\hline PARK & 0.910 & kstar-galaxy classification parameter \\
\hline PARMU & 0.187 & mu star-galaxy classification parameter \\
\hline IGAL & 1 & final classification flag (1 for a galaxy) \\
\hline JON & -1 & eyeball classification flag \\
\hline ORIENT & 91.0 & orientation in degrees clockwise from E-W \\
\hline ECCENT & 0.270 & eccentricity \\
\hline AREA & 308.0 & isophotal area in pixels \\
\hline $\mathrm{X}_{-} \mathrm{BJ}$ & 2918.7 & plate $\mathrm{x}_{\mathrm{b}}$ in 8 micronpixels \\
\hline $\mathrm{Y}_{-}^{-} \mathrm{BJ}$ & 9123.1 & plate $y_{b_{j}}$ in 8 micronpixels \\
\hline $\mathrm{D} \overline{\mathrm{X}}$ & 43.0 & corrected difference $100\left(\mathrm{x}_{\mathrm{b}_{J}}-\mathrm{x}_{\mathrm{R}}\right)$ \\
\hline DY & 49.0 & corrected difference $100\left(\mathrm{y}_{\mathrm{b}_{J}}-\mathrm{y}_{\mathrm{R}}\right)$ \\
\hline BJG & 18.99 & bj without extinction correction \\
\hline RMAG & 10.35 & unmatched APM 'total' mag \\
\hline PMAG & 10.53 & unmatched raw APM profile integrated mag \\
\hline FMAG & 8.72 & unmatched raw APM 2" 'fibre' mag \\
\hline SMAG & 10.74 & unmatched raw stellar mag (from APMCAL) \\
\hline IFIELD & 417 & UKST field \\
\hline IGFIELD & 2007 & galaxy number in UKST field \\
\hline REGION & 'S417' & GSSS region name \\
\hline OBJEQNX & 2000.00 & equinox of the plate reference frame \\
\hline OBJRA & 0.8034094522 & RA (J2000) in radians : 030407.673 \\
\hline OBJDEC & -0.5441390223 & DEC (J2000) in radians: -311036.73 \\
\hline PLTSCALE & 67.2000 & plate scale in arcsec per mm \\
\hline XPIXELSZ & 25.2844500 & Xpixel size in microns \\
\hline YPIXELSZ & 25.2844500 & Y pixel size in microns \\
\hline OBJPLTX & 7970.86 & object $x$ on plate (pixels) \\
\hline OBJPLTY & 4148.11 & object Y on plate (pixels) \\
\hline DATAMAX & 14431 & maximum data value \\
\hline DATAMIN & 4011 & minimum data value \\
\hline BJSELOLD & 18.96 & original bj mag used in the object selection \\
\hline BJG_OLD & 19.01 & original bj without extinction correction \\
\hline END & & End of FITS header \\
\hline
\end{tabular}


Table 4. FITS keywords for the spectra (extensions $1 . .$. spectra).

\begin{tabular}{|c|c|c|}
\hline Keyword & Example & Definition \\
\hline XTENSION & 'IMAGE' & IMAGE extension \\
\hline BITPIX & -32 & number of bits per data pixel \\
\hline NAXIS & 2 & number of data axes \\
\hline NAXIS 1 & 1024 & length of data axis 1 \\
\hline NAXIS2 & 3 & length of data axis 2 \\
\hline PCOUNT & 0 & required keyword; must $=0$ \\
\hline GCOUNT & 1 & required keyword; must=1 \\
\hline CRVAL1 & 5802.8979492 & coordinate value of axis 1 \\
\hline CDELT1 & 4.3103027344 & coordinate increment on axis 1 \\
\hline CRPIX1 & 512.0000000000 & reference pixel on axis 1 \\
\hline CUNIT1 & 'Angstroms' & units for axis 1 \\
\hline EXTNAME & 'SPECTRUM' & 2 dFGRS spectrum \\
\hline OBSNAME & 'TGS4697164' & observed object name \\
\hline OBSRA & 0.7943429758 & observed RA (B1950) in radians \\
\hline OBSDEC & -0.5475286940 & observed DEC (B1950) in radians \\
\hline MATCH_DR & 0.0000 & position match error in arcsec \\
\hline $\mathrm{Z}-\mathrm{-}$ & 0.178876 & raw measured redshift \\
\hline Z_HELIO & 0.178860 & heliocentric redshift \\
\hline QÜALITY & 5 & redshift measurement quality \\
\hline ABEMMA & 1 & redshift type: $a b s=1, e m i=2, \operatorname{man}=3$ \\
\hline NMBEST & 0 & \# emission lines for emission z \\
\hline NGOOD & 0 & number of good emission lines \\
\hline Z_EMI & -9.9990 & emission redshift \\
\hline Q_Z_EMI & 0 & emission redshift quality \\
\hline$\overline{K B E S T R}$ & 2 & cross-correlation template \\
\hline R_CRCOR & 15.5600 & cross-correlation peak \\
\hline $\mathrm{Z}_{-} \mathrm{ABS}$ & 0.1789 & cross-correlation redshift \\
\hline$Q_{-}^{-} Z \_A B S$ & 3 & cross-correlation quality \\
\hline Q_EINAL & 3 & suggested quality for redshift \\
\hline IĀLTER & 0 & IALTER=1 if automatic $\mathrm{z}$ altered \\
\hline Z_COMM & $1 '$ & observer's comment \\
\hline THPUT & 0.96613 & fibre throughput \\
\hline SPFILE & 'sgp469_991104_1z.fits' & $2 d F$ reduced data file \\
\hline PLATE & 1 & $2 \mathrm{dF}$ plate number \\
\hline PIVOT & 302 & $2 \mathrm{dF}$ pivot number \\
\hline FIBRE & 58 & $2 \mathrm{dF}$ fibre number \\
\hline OBSRUN & '990CT' & observation run \\
\hline GRS_DATE & '991104' & $2 \mathrm{dF}$ YYMMDD observed date \\
\hline UTDĀTE & '1999:11:04' & UT date of observation \\
\hline SPECTID & 'A' & $2 d F$ spectrograph ID \\
\hline GRATID & $' 300 B^{\prime}$ & $2 \mathrm{dF}$ grating ID \\
\hline GRATLPMM & 300 & $2 \mathrm{dF}$ grating line per mm \\
\hline GRATBLAZ & 'COLLIMATOR' & 2dF grating blaze direction \\
\hline GRATANGL & 25.30000 & $2 d F$ grating angle \\
\hline LAMBDAC & 5782.700 & central wavelength \\
\hline $\mathrm{CCD}$ & 'TEKTRONIX_5' & CCD ID \\
\hline CCDGAIN & $2 . \overline{7} 90$ & CCD inverse gain (e per ADU) \\
\hline CCDNOISE & 5.200 & CCD read noise (electrons) \\
\hline OBJX & 196833 & $2 \mathrm{dF}$ object $\mathrm{X}$ position \\
\hline OBJY & 10401 & 2dF object Y position \\
\hline OBJXERR & 6 & $2 \mathrm{dF}$ object $\mathrm{X}$ position error \\
\hline OBJYERR & 14 & $2 \mathrm{dF}$ object y position error \\
\hline OBJMAG & 18.96 & $2 \mathrm{dF}$ object magnitude \\
\hline THETA & 4.526 & $2 \mathrm{dF}$ fibre angle \\
\hline PTRTYPE & $' \mathrm{P}^{\prime}$ & $2 d F$ ptrtype \\
\hline PID & 0 & $2 d F$ pid \\
\hline OBSELD & 'sgp469' & $2 \mathrm{dF}$ observed field number \\
\hline $\mathrm{NCOMB}$ & 3 & number of frames combined \\
\hline REFRUN & 31 & AAT run number of reference \\
\hline UTSTART & $' 16: 37: 59.48 '$ & UT start of reference exposure \\
\hline UTEND & $' 16: 57: 59 \prime$ & UT end of reference exposure \\
\hline REFEXP & 1200.0 & reference run exposure (secs) \\
\hline REFHASTA & $' 36.07264^{\prime}$ & HA start of reference exposure \\
\hline REFHAEND & $' 41.08119 '$ & HA end of reference exposure \\
\hline ETA_TYPE & $-2.5934501 \mathrm{E}+00$ & eta spectral type parameter \\
\hline SNR & $2.0299999 \mathrm{E}+01$ & median S/N per pixel \\
\hline
\end{tabular}


Table 5. Additional mSQL data base keywords.

\begin{tabular}{|c|c|c|}
\hline \multicolumn{3}{|c|}{ (i) Keywords in all extensions } \\
\hline Keyword & Example & Definition \\
\hline serial & 100100 & 2 dFGRS serial number \\
\hline name & TGS469Z164 & $2 \mathrm{dFGRS}$ name \\
\hline UKST & 417 & UKST sky survey field number \\
\hline spectra & 1 & number of spectra for this object \\
\hline extnum & 1 & extension number \\
\hline obsrun & 990CT & observing run year and month \\
\hline TDFgg & -469 & $2 \mathrm{dFGRS}$ field number (+NGP, $-\mathrm{SGP})$ \\
\hline pivot & 302 & $2 \mathrm{dF}$ pivot \\
\hline plate & 1 & $2 \mathrm{dF}$ plate \\
\hline fiber & 58 & $2 \mathrm{dF}$ fibre \\
\hline z & 0.178876 & observed redshift \\
\hline z_helio & 0.178860 & heliocentric redshift \\
\hline a bemma & 1 & redshift type $(a b s=1, e m i=2, \operatorname{man}=3)$ \\
\hline quality & 5 & redshift quality parameter \\
\hline \multicolumn{3}{|c|}{ (ii) Keywords in extension 0 only } \\
\hline Keyword & Example & Definition \\
\hline alpha & 0.7943429758 & RA (B1950) in radians \\
\hline delta & -0.5475286941 & $\mathrm{DEC}$ (B1950) in radians \\
\hline ra & 323.00 & RA (B1950) in HH MM SS.SS \\
\hline dec & -312215.9 & DEC (B1950) in DD MM SS.S \\
\hline ra2000 & 030407.68 & RA (J2000) in HH MM SS.SS \\
\hline $\operatorname{dec} 2000$ & -311036.8 & DEC (J2000) in DD MM SS.S \\
\hline 12 & 228.9258834424 & Galactic longitude \\
\hline b2 & -60.8572447739 & Galactic latitude \\
\hline
\end{tabular}

(extension 0 ) of the FITS files; Table 4 lists all the keywords present in the spectrum extensions ( $1 \ldots$ spectra). These tables give the names of the keywords, example values, and the keyword definitions.

\section{2 $\mathrm{mSQ} L$ parameter data base}

The mSQL data base can be thought of as a table. The rows of the table are labelled by the unique object serial number ( $S E Q N U M$ ) and the extension number (extnum), with multiple rows for each target object corresponding to each extension in the object's FITS file. The columns of the table correspond to the object parameters, and are labelled by the name of the corresponding keyword. The object serial numbers (SEQNUM) provide the primary data base key, but the objects are also indexed by their unique survey name (NAME), which has the format TGhfffznnn, where $h$ is the hemisphere (N for the NGP strip and $S$ for the SGP strip and random fields); $\mathrm{ff} f$ is the number of the primary field to which the object is assigned, and $n n n$ is the number of the galaxy within that field. Note that the observed name of the object (parameter OBSNAME in each spectrum extension) is the same as NAME except that (i) if the field in which the object is observed (given by OBSFLD) is an overlapping field rather than its primary field (given by $f f f$ ), then the first character of the name is changed from $T$ to $\mathrm{X}$, and (ii) if the object has been flagged as a possible merger, then the second character of the name is changed from $\mathrm{G}$ to $\mathrm{M}$.

The first row for each object (extnum $=0$ ) contains the source catalogue data and the basic spectroscopic information from the best spectrum of that object. The keywords for that row are the FITS parameters for the primary image (Table 3) plus all the additional keywords listed in Table 5 . The best spectrum is the one with the highest redshift quality parameter; if there is more than one spectrum of the same quality, then the most recent of these spectra is used. Subsequent rows for the same object (extnum $=1 \ldots$ spectra, where spectra is the number of spectra obtained for that object) contain the FITS parameters pertaining to each spectroscopic observation (Table 4) plus the additional keywords in section (i) of Table 5. If there is no spectrum for the object, then spectra $=0$ and only the row corresponding to extnum $=0$ will exist. Note that some information is duplicated between rows and that not all parameters are defined for all rows; undefined parameters return a NULL value.

Searches of the data base use the mSQ L query format (J epson \& Hughes 1998), which has the basic format

SELECT list_of_parameters FROM database_name WHERE list_of_conditions

and list_of_conditions is a series of equalities and inequalities linked by Boolean relations.

An example is

SELECT name, extnum, ra, dec, BJSEL, Z, QUALITY, $z$, quality FROM public WHERE name = 'TGS4697164'

which selects the listed parameters for the object with $2 \mathrm{dFGRS}$ name TGS 4697164 (note the single quotes around the character string) from both the summary row (extnum $=0$ ) and for each spectrum (extnum $=1$...spectra). Note that the parameters with the same name in lower case and upper case are distinct: the former are generally from extnum $=0$, and the latter from extnum $>0$ (parameters are returned as NULL in rows where they are not defined).

An example with a more complex list of conditions is

SELECT name FROM public WHERE extnum = 0 AND ( (BJSEL < 15.5 AND quality > =3) oR quality > 4)

which lists just the names of the objects which are either brighter than $b_{j}=15.5$ with redshift quality at least 3 , or have quality greater than 4 , or both; the search is restricted just to the summary row by requiring extnum $=0$. 
Simple searches on the two indexed parameters, serial and name), are quick - e.g., WHERE serial=69656 or WHERE name = 'TGS203Z081'; more complex searches take about 5 minutes. Further information about the $\mathrm{MSQL}$ data base software and its structured query language is given in Yarger et al. (1999) and on the WW W at http: / / www. hughes. com. au.

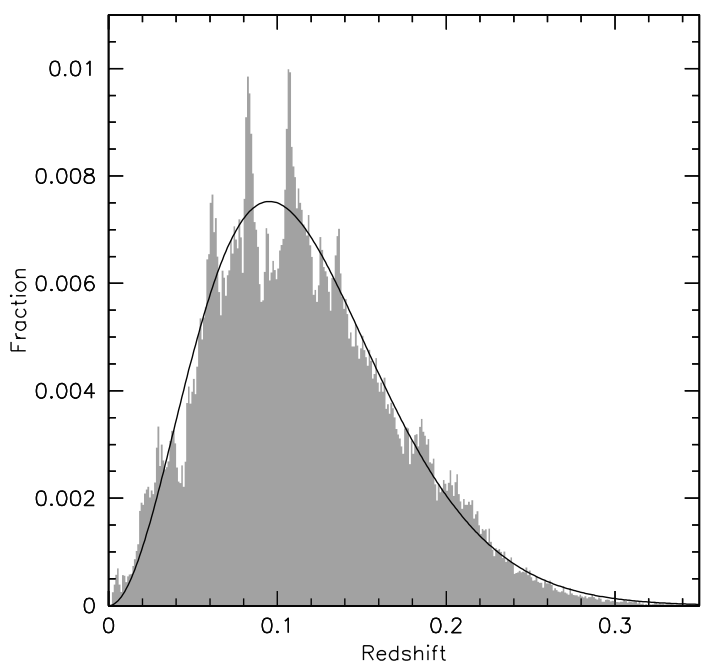

Figure 17. The redshift distribution of the 2dFGRS (histogram) and a simple smooth analytic approximation (curve).

\subsection{WWW interface}

The $2 \mathrm{dFGRS} \mathrm{mSQL}$ data base can be searched via the WWW interface in a number of ways: (i) search via a standard $\mathrm{mSQL}$ query as described above - this is the most general method; (ii) perform a standard $\mathrm{mSQL}$ query restricted to a list of named objects; (iii) perform a standard $\mathrm{mSQL}$ query restricted to objects within a specified radius of a given position on the sky, and (iv) match objects to a supplied catalogue of positions.

The results of a query can be returned in several forms: as an HTM L table, as an ASCII table, as a gzipped ASCII file, as an e-mail giving the URL of a gzipped ASCII file created with a background job, or as a tar file containing the FITS files for the selected objects. The HTML table has buttons allowing one to select a particular object and view the postage stamp DSS image and all the observed spectra for the object; if the spectra have measured redshifts, then the positions of prominent spectral features are indicated at the redshift associated with each individual spectrum. The FITS files for the objects in the HTM L table can also be bundled up into a compressed tar file which can then be downloaded by anonymous ftp.

Full instructions for accessing the data base are given on the survey website at http: / / www. mso. anu.edu.au/2dFGRS and at mirror sites given there.

\section{RESULTS}

The redshift distribution of the galaxies in the survey is shown in Fig. 17. Also shown is a simple analytic approximation to the

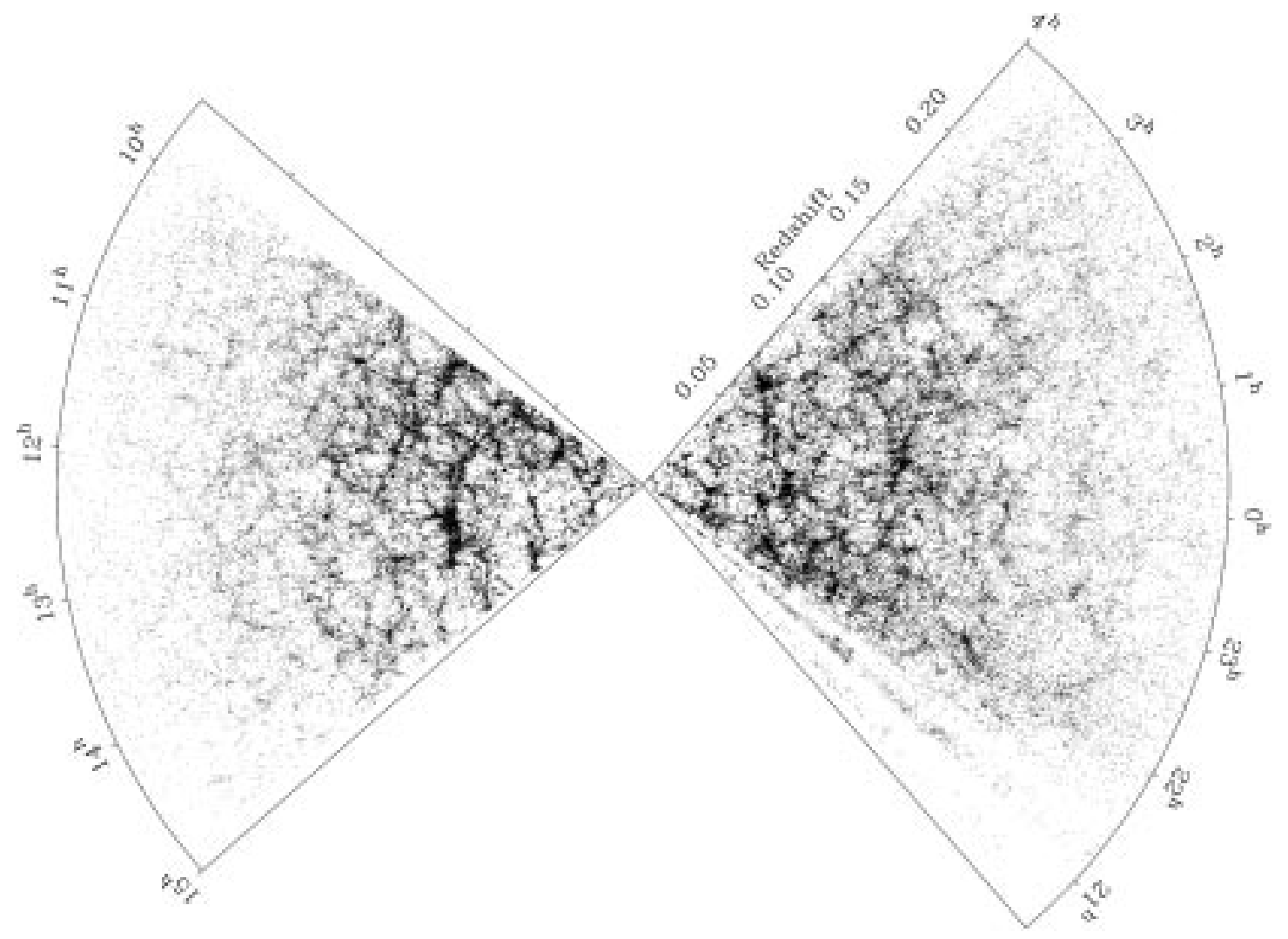

Figure 18. The projected distribution of the galaxies in the NGP (top) and SGP (bottom) strips, as a function of redshift and RA; the variations in the galaxy density with RA are due to variations in the effective widths of the strips. 

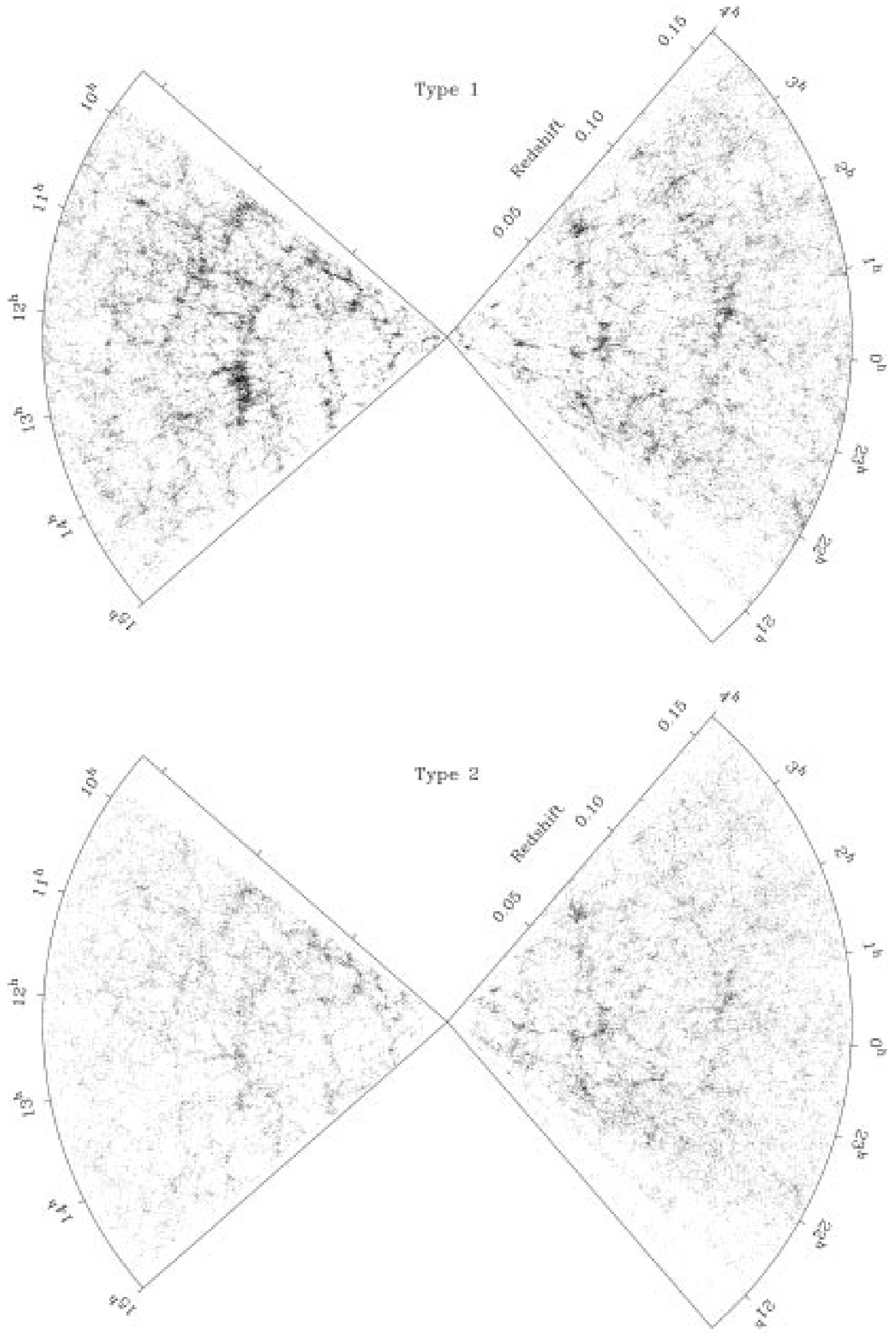

Figure 19. Redshift slices for different spectral types: type 1 corresponds to E/SO, type 2 to Sa/Sb, type 3 to Sc/Sd and type 4 to Irr. 
2dFGRS: spectra and redshifts
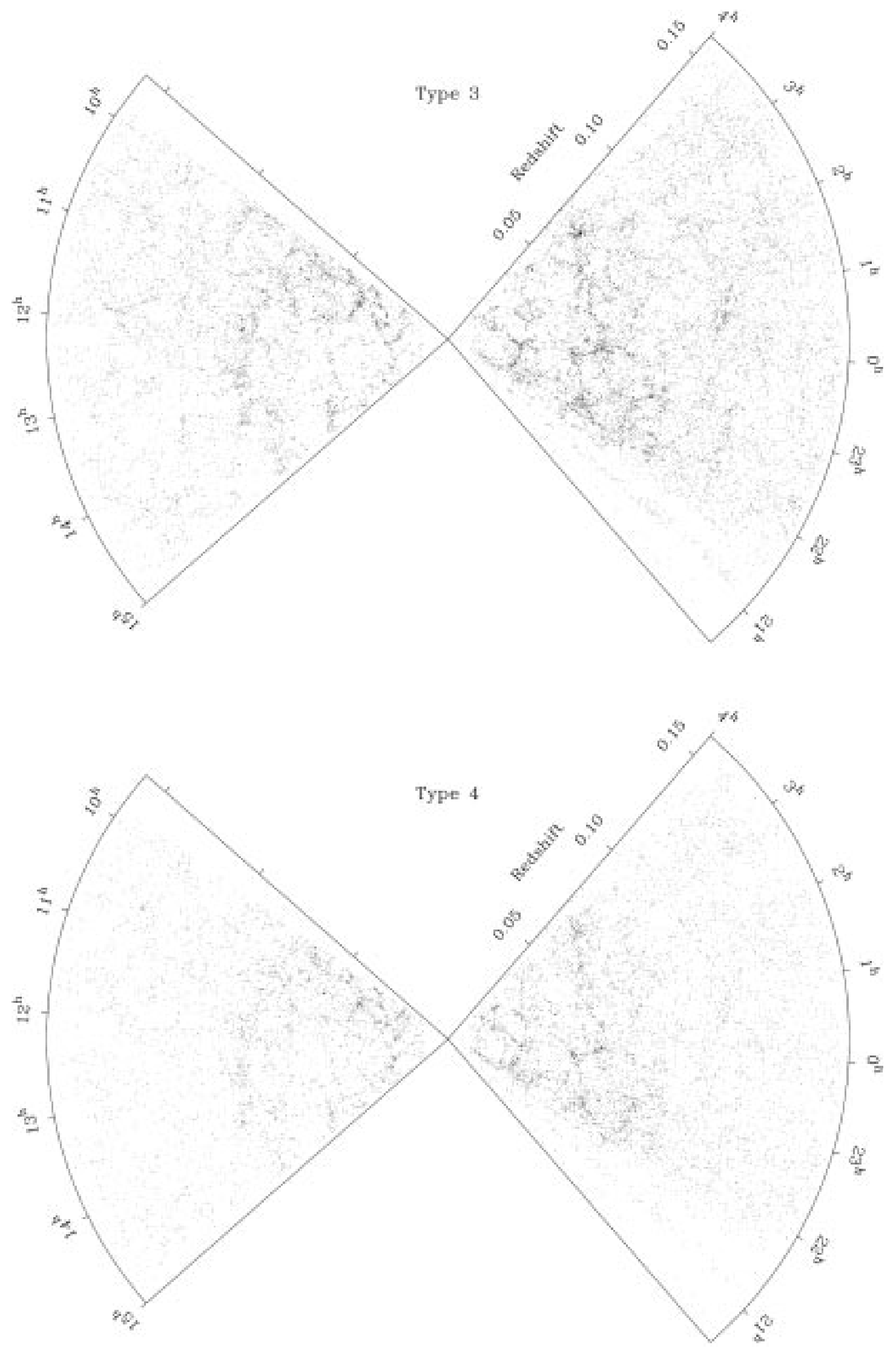

Figure 19 - continued 
redshift distribution, following Efstathiou \& M oody (2001), with the form

$d N \propto z^{2} \exp \left[-\left(\frac{1.36 z}{z}\right)^{1.55}\right] d z$,

where $z=0.11$ is the median redshift of the survey.

The spatial distribution of the galaxies in the survey strips is shown in Fig. 18. This figure is the projection of the full width of the strips ( $10^{\circ}$ in the NGP and $15^{\circ}$ in the SGP). A s not all fields have yet been observed, there are variations in the effective thicknesses of the strips in the directions of the missing fields that produce corresponding variations in the galaxy density. The clustering of galaxies revealed in this figure has been investigated by Peacock et al. (2001); Percival et al. (2001) and Norberg et al. (2001).

The 2dFGRS has provided the first clear detection of the redshift-space clustering anisotropy on large scales that is a key prediction of the gravitational instability paradigm for the growth of structure in the U niverse (Peacock et al. 2001). M easurements of this distortion yield a precise estimate of the parameter $\beta=$ $\Omega^{0.6} / b=0.43 \pm 0.07$, where $\Omega$ is the total mass density of the $U$ niverse, and $b$ is the linear bias of the galaxies with respect to the mass.

The power spectrum of the galaxy distribution has been determined from the survey using a direct FFT-based technique (Percival et al. 2001). Over the range in wavenumber $0.02<\mathrm{k}<0.15 \mathrm{~h} \mathrm{M} \mathrm{pc}^{-1}$, the shape of the observed power spectrum will be close to that of the linear density perturbations convolved with the window function of the survey. Fitting convolved power spectrum models constrains the shape parameter $\Gamma=\Omega \mathrm{h}$ to be $0.20 \pm 0.03$ (68 per cent confidence level), and shows that models containing baryon oscillations are preferred over models without baryons at the 95 per cent confidence level. This is the first detection of baryon oscillations in the galaxy distribution, and yields an estimate for the baryon fraction $\Omega_{\mathrm{b}} / \Omega_{\mathrm{m}}=0.15 \pm 0.07$, assuming scale-invariant primordial fluctuations.

Combined with recent measurements of the anisotropies in the $C M B$, the clustering in the survey provides strong evidence for a low-density $\left(\Omega_{\mathrm{m}} \approx 0.3\right)$ Universe with a cosmological constant $\left(\Omega_{\Lambda} \approx 0.7\right)$ - see Efstathiou et al. (2001).

The size of the $2 \mathrm{dF} G R S$ sample has allowed investigation of the variation in the strength of galaxy clustering with luminosity, using the projected two-point correlation function of galaxies in a series of volume-limited samples (N orberg et al. 2001). The clustering of $L^{*}\left(M_{b_{1}}^{*}-5 \log h=-19.7\right)$ galaxies in real space is well fitted by a power-law relation with exponent $\gamma=1.71 \pm 0.06$ and $a$ correlation length $r_{0}^{*}=(4.9 \pm 0.3) \mathrm{h}^{-1} \mathrm{M} \mathrm{pc}$. The exponent shows very little variation for galaxy samples with luminosities differing by a factor of 40 . The clustering amplitude, however, increases with luminosity: slowly for galaxies fainter than $M *$, but more strongly at brighter absolute magnitudes. This dependence of the correlation length on luminosity is in good agreement with the predictions of the hierarchical galaxy formation models of B enson et al. (2001). In terms of the bias parameter, the relation is well represented by $\delta b / b^{*}=0.15 \delta L / L *$, where $\delta b=b-b^{*}$, $\delta \mathrm{L}=\mathrm{L}-\mathrm{L}^{*}$, and the relative bias is given by $b / b^{*}=\left(r_{0} / r_{0}^{*}\right)^{\gamma / 2}$.

The 2dFGRS has also been used to characterize the internal properties of the galaxy population. Folkes et al. (1999) and M adgwick et al. (2001a) have determined the luminosity function for galaxies, both overall and as a function of spectral type. Principal component analysis has been applied to the 2dFGRS spectra, and a linear combination of the first two principal components, $\eta$, has been used to parametrize the spectral type. Going from early types to late types, the luminosity functions appear to exhibit a systematic decrease in the characteristic luminosity (from $M_{b_{1}}^{*}-5 \log h=-19.6$ to -19.0 ) and $a$ steepening of the faint-end slope (from $\alpha=-0.52$ to -1.43 ). However, there is also evidence that, at the precision afforded by the $2 \mathrm{dFGRS}$ sample, the standard Schechter fitting function is no longer an adequate representation of the luminosity function.

Fig. 19 shows the redshift slices for each of the four spectral types defined in terms of the $\eta$ parameter by Madgwick et al. (2001a): type 1 are the earliest types, and type 4 the latest types. The association of earlier types with clusters and local density enhancements is apparent, as is the tendency for later types to be associated with lower density regions. A quantitative study of the clustering of different spectral types will be the subject of a subsequent paper.

The large $2 \mathrm{dF}$ GRS sample also allows the generalization of the luminosity function into the bivariate brightness distribution (BBD) over both luminosity and surface brightness (Cross et al. 2001). The BBD derived from the $2 \mathrm{dFGRS}$ shows a strong surface brightness - luminosity relation, $M_{b_{j}} \propto\left(2.4 \pm_{0.5}^{1.5}\right) \mu_{e}$. The luminosity density is dominated by normal giant galaxies and the peak of the B BD lies away from the survey selection boundaries, implying that the $2 \mathrm{dFGRS}$ is complete and that luminous low surface brightness galaxies are rare. By integrating over the $B B D$, the local luminosity density is estimated to be $j_{B}=(2.5 \pm 0.2) \times 10^{8} \mathrm{hL}_{\odot} \mathrm{M} \mathrm{pc}^{-3}$.

Cole et al. (2001) have combined the 2dFGRS with the 2M ASS extended source catalogue to produce an infrared-selected sample of over 17000 galaxies with redshifts. This sample has been used to determine the $\mathrm{J}$ and $\mathrm{K}_{\mathrm{S}}$-band galaxy luminosity functions. The luminosity functions (in 2M A SS K ron magnitudes) are fairly well fitted by Schechter functions: in J with $M_{1}^{*}-5 \log h=-22.36 \pm$ $0.02, \alpha_{J}=-0.93 \pm 0.04, \Phi_{\jmath}^{*}=0.0104 \pm 0.0016 \mathrm{~h}^{3} \mathrm{M} \mathrm{pc}^{-3}$, and in $_{*} K_{S}$ by $M_{K}^{*}-5 \log h=-23.44 \pm 0.03, \alpha_{K}=-0.96 \pm 0.05$, $\Phi_{\mathrm{K}}^{*}=0.0108 \pm 0.0016 \mathrm{~h}^{3} \mathrm{M} \mathrm{pc}^{-3}$. F rom the distributions of $\mathrm{B}-\mathrm{K}$ and $J-K$ colours with absolute magnitude and models of the stellar populations, the galaxy stellar-mass function can be estimated. Integrated over all galaxy masses, it yields a total mass fraction in stars (in units of the critical density) of $\Omega_{\text {stars }} h=(1.6 \pm 0.2) \times 10^{-3}$ for a Kennicutt IMF, and $\Omega_{\text {stars }} h=(2.9 \pm 0.4) \times 10^{-3}$ for a Salpeter IM F. These values are consistent with estimates based on the time integral of the observed star formation history of the Universe only if dust extinction corrections at high redshift are modest.

The 2dFGRS can add significant value to all-sky imaging surveys at all wavelengths, as in the studies of the radio galaxy population combining redshifts and optical spectra from the 2dFGRS with the NVSS radio survey (Sadler et al. 2001) and the FIRST radio survey ( $M$ agliocchetti et al. 2001). Using 20 per cent of the full 2dFGRS area, Sadler et al. find 757 optical counterparts for NVSS sources - the largest and most homogeneous set of radio-source spectra to date. These sources range from $z=0.005$ to $z=0.438$, and are a mixture of active galaxies ( 60 per cent) and star-forming galaxies (40 per cent). The local radio luminosity function at $1.4 \mathrm{GHz}$ is determined for both active and star-forming galaxies, and yields an estimate for the local star formation density of $(2.8 \pm 0.5) \times 10^{-3} \mathrm{M}_{\odot} \mathrm{yr}^{-1} \mathrm{~h}^{3} \mathrm{M} \mathrm{pc}^{-3}$.

\section{CONCLUSIONS}

The 2dF Galaxy Redshift Survey (2dFGRS) is designed to measure 
redshifts for approximately 250000 galaxies. The survey uses the $2 \mathrm{dF}$ multifibre spectrograph on the Anglo-A ustralian Telescope, which is capable of observing up to 400 objects simultaneously over a $2^{\circ}$ diameter field of view. The source catalogue for the survey is a revised and extended version of the APM galaxy catal ogue, which is based on A utomated Plate M easuring machine (APM) scans of 390 plates from the UK Schmidt Telescope (UK ST) Southern Sky Survey. The target galaxies have extinctioncorrected magnitudes brighter than $b_{j}=19.45$, with extinctions derived from the maps of Schlegel et al. (1998).

The main survey regions are two contiguous declination strips, one in the southern Galactic hemisphere (the SG P strip) covering $80^{\circ} \times 15^{\circ}$ centred close to the $\mathrm{SGP}$, and the other in the northern Galactic hemisphere (the NGP strip) covering $75^{\circ} \times 10^{\circ}$. In addition, there are 99 random fields are spread uniformly over the entire region of the APM catalogue in the southern Galactic hemisphere outside the SGP strip. In total the survey covers some $2000 \mathrm{deg}^{2}$ and has a median depth of $z=0.11$. O ut to the effective limit of the survey at $z \approx 0.3$, the strips contain a volume of $1.2 \times 10^{8} \mathrm{~h}^{-3} \mathrm{M} \mathrm{pc}^{3}$; the volume sparsely sampled including the random fields is 2 to 3 times larger. A $n$ adaptive tiling al gorithm is used to give a highly uniform sampling rate of 93 per cent over the whole survey region.

Spectra are obtained over the wavelength range $3600-8000 \AA$ at two-pixel resolution of $9.0 \AA$. The median $S / N$ is 13 pixel $^{-1}$ over 4000-7500 $\AA$. Redshifts are measured both by cross-correlation with a range of template spectra and by fitting strong spectral features. All redshift identifications are visually checked and assigned a quality parameter $Q$ in the range 1-5. From repeat measurements and comparisons with other redshift catalogues, we find that redshifts with $Q=3$ have a blunder rate (fraction of incorrect identifications) of just under 10 per cent, and $Q=4$ and 5 redshifts have blunder rates less than 1 per cent - the overall blunder rate for reliable $(Q \geq 3)$ redshifts is 3 per cent. The overall rms uncertainty in the $Q \geq 3$ redshifts is $85 \mathrm{~km} \mathrm{~s}^{-1}$. The completeness of the survey is computed as a function of both field and apparent magnitude. The overall redshift completeness is 91.8 per cent, but this varies with magnitude from 99 per cent for the brightest galaxies to 90 per cent for objects at the survey limit.

The survey data base has two components: a collection of FITS files, one per object, which contain all the parameters and spectra for each object, and a mSQL parameter data base which can be used for sophisticated searching, matching and sorting of the survey data. The $2 \mathrm{dF} G R S$ data base is available through the survey WWW site at http://WwW.mso.anu.edu.au/2dFGRS.

\section{ACKNOWLEDGMENTS}

The $2 \mathrm{dF}$ Galaxy Redshift Survey was made possible through the dedicated efforts of the staff of the Anglo-A ustralian Observatory, both in creating the $2 \mathrm{dF}$ instrument and in supporting the survey observations.

\section{REFERENCES}

Arnouts $S$. et al., 2001, $A \& A$, in press

Bailey J. A., Glazebrook K., Offer A., Taylor K., 2001, M NRAS, in press

Benson A. J., Frenk C. S., Baugh C. M., Cole S., Lacey C. G., 2001, MNRAS, 327, 1041

Blair M., Gilmore G., 1982, PASP, 94, 741

B oyle B. J., Shanks T., Croom S. M., Smith R. J., Miller L., Loaring N., Heymans C., 2000, MNRAS, 317, 1014

Cole S. et al. (2dFGRS team), 2001, MNRAS, 326, 255

Colless M. M., 1999, Phil. Trans. Roy. Soc. Lond. A, 357, 105

Croom S. M., Smith R. J., Boyle B. J., Shanks T., L oaring N. S., M iller L., Lewis I. J., 2001, M NRAS, 322, 29

Cross N. et al. (2dFGRS team), 2001, MNRAS, 324, 825

De Propris R. et al. (2dFGRS team), 2001, M NRAS, in press

Efstathiou G., M oody S. J., 2001, M NRAS, 325, 1603

Efstathiou G. et al. (2dFGRS team), 2001, M NRAS, submitted

Folkes S. et al. (2dFGRS team), 1999, MNRAS, 308, 459

Huchra J. P., Vogeley M. S., Geller M. J., 1999, A pJ S, 121, 287

Jacoby G. H., Hunter D. A., Christian C. A., 1984, A pJ S, 56, 257

Jaffe A. H. et al., 2001, Phys. Rev. Lett., 86, 3475

Jepson B., Hughes D. J., 1998, The Official Guide to miniSQ L 2.0. John Wiley \& Sons, Brisbane

Kennicutt R. C., 1992, A pJ S, 79, 255

Lasker B. M. et al., 1998, BA AS, 192, 6403

Lewis I. J. et al., 2001, in press

L oveday J., M addox S. J., Efstathiou G., Peterson B. A., 1996, A pJ S, 107, 201

M addox S. J., Efstathiou G., Sutherland W. J., L oveday J., 1990a, M NRAS, 242, 43p

M addox S. J., Efstathiou G., Sutherland W. J., L oveday J., 1990b, M NRA S, 243, 692

M addox S. J., Efstathiou G., Sutherland W. J., 1990c, M NRA S, 246, 433

M addox S. J., Efstathiou G., Sutherland W. J., 1996, M NRAS, 283, 1227

Madgwick D. S. et al. (2dFGRS team), 2001a, M NRAS, submitted

Madgwick D. S. et al. (2dFGRS team), 2001b, in Banday A. et al., ed., M ining the Sky. ESO A strophysics Symposia. Springer-Verlag, in press

M agliochhetti M. et al. (2dFGRS team), 2001, M NRAS, submitted

M etcalfe N., Fong R., Shanks T., 1995, M NRAS, 274, 769

M onet D. et al., 1996, USN O-A 1.0: A Catalog of A strometric Standards. US Naval Obs., Washington

Norberg P. et al. (2dFGRS team), 2001a, M NRAS, 328, 67

Norberg P. et al. (2dFGRS team), 2001b, M NRAS, submitted

Peacock J. A. et al. (2dFGRS team), 2001, Nat, 410, 169

Percival W. J. et al. (2dFGRS team), 2001, M NRAS, submitted

Pimbblet K. A., Smail I., Edge A. C., Couch W. J., O'Hely E., Zabludoff A. I., 2001, M NRAS, 327, 588

Sadler E. M . et al. (2dFGRS team), 2001, M NRAS, submitted

Saunders W. et al., 2000, M NRAS, 317, 55

Schlegel D. J., Finkbeiner D. P., Davis M., 1998, A pJ, 500, 525

Shectman S. A., Landy S. D., Oemler A., Tucker D. L., Lin H., K irshner R. P., Schechter P. L., 1996, A pJ , 470, 172

Tonry J. L., Davis M., 1979, AJ, 84, 1511

Yarger R. J., Reese G., King T., 1999, MySQL and mSQL, O'Reilly \& Associates

This paper has been typeset from a $\mathrm{T}_{\mathrm{E}} \mathrm{X} / \mathrm{LT}_{\mathrm{E}} \mathrm{X}$ file prepared by the author. 